\title{
Solving the Performance Puzzle of DSRC Multi-Channel Operations
}

\author{
Kai Xiong
}

School of Computer Science

McGill University

Montreal, Canada

August 2014

A thesis submitted to McGill University in partial fulfillment of the requirements for the degree of Master of Science.

(C) 2014 Kai Xiong 


\section{Dedication}

This thesis is dedicated to my parents, my sister, my love and the whole research community here at McGill University 


\begin{abstract}
The IEEE 802.11p based Dedicated Short Range Communication (DSRC) protocol is a key enabling technology for enhancing road safety and transportation efficiency, and is being seriously considered by major research centres and automobile manufacturers. Wireless Access in Vehicular Environments (WAVE) 1609.4 is a new amendment that enables multi-channel operations in DSRC. Operating intervals are divided into alternating Control Channel $(\mathrm{CCH})$ Intervals and Service Channel (SCH) Intervals, which halve the duration of channel intervals and operating interval. This alternating feature causes high packet losses in $\mathrm{CCH}$ and low throughput in $\mathrm{SCH}$, hence hinders the deployment of this protocol. The key goal of our work is to provision sufficient reliability for real-time safety messages in $\mathrm{CCH}$ while optimising non-safety service delivery in SCH. We have developed analytical models for both broadcasting and unicasting to explore the relationship among traffic density, $\mathrm{CCH}$ packet loss ratio, SCH throughput, and the duration of each kind of intervals. Unlike assigning fixed channel interval in error-prone default channel access approach, we develop a multi-channel coordination algorithm which adaptively adjusts the duration of intervals to achieve better performance and reliability based on these models. Theoretical analysis and extensive simulation results demonstrate the accuracy of our model and the efficacy of the proposed algorithm. The algorithm almost doubles SCH throughput in low traffic density scenario and reduces packet loss ratio by up to $30 \%$. We also show that the proposed algorithm converges rapidly to the optimal channel interval division.
\end{abstract}




\section{ABRÉGÉ}

Le protocole IEEE 802.11p dédié à la communication sur des distances courtes (DSRC) est présentement considéré par les centres de recherches et les centres manufacturiers d'automobiles comme un protocole améliorant la sécurité routiàre ainsi que l'efficacité du transport routier. Le protocole WAVE 1609.4 constitue un nouvel amendement envers DSRC permettant les opérations multicanaux lorsque l'intervalle de fonctionnement est divisé en alternance entre les intervalles du canal de contrôle $(\mathrm{CCH})$ et du canal de service (SCH). La bande passante de chaque canal est donc divisée par deux. Le but de nos travaux est de fournir suffisamment de fiabilité en temps réel pour les messages ayant trait à la sûreté du canal de contrôle tout en optimisant les services du canal de service ( $\mathrm{SCH}$ ). Contrairement à l'approche qui attribue un intervalle statique pour le canal d'erreur par défaut, nous dveloppons un algorithme dynamique d'ajustement du canal basé sur la théorie itérative du contrôle afin de résoudre le problème. Nous développons des modèles pour la diffusion à large échelle et la diffusion unilatérale afin d'explorer les interconnexions entre la densité du trafic (congestion) et le ratio de la perte de paquets. De plus, nous discutons de l'importance de choisir un intervalle optimal pour le canal et nous apportons un éclairage nouveau concernant le choix adaptatif d'une approche par accès de canaux qui maximise l'utilisation du canal. Des analyses thoriques exhaustives et des simulations démontrent que nos modèles sont précis, que l'algorithme est efficace et converge vers la solution optimale. 


\section{Acknowledgments}

First and foremost, I would like to express my sincere gratitude to my supervisor, professor Xue Liu, for his tremendous support of my master study and research, for his patience,

immense knowledge and great research vision. His guidance helped me all the time of research and writing of this thesis.

Many thanks go to Dr. Lei Rao, for her time to organize weekly meeting to discuss this project and her constructive and insightful comments on my work.

I thank members in the Cyber-Physical Systems lab, especially Xi Chen, Yuan Yao and Chu Wang. The positive research atmosphere leads to friendly collaboration, discussion and great work efficiency. Special thanks go to Claude Gravel and Javona White Bear for helping me proofread this thesis.

Last but not the least, I would like to thank my parents Donglin Xiong and Zhiying $\mathrm{Xu}$, for giving birth to me at the first place and supporting me spiritually throughout my life. 


\section{Contents}

1 Introduction $\quad 1$

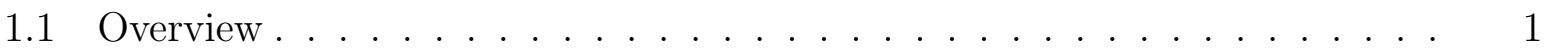

1.2 Problem Definition . . . . . . . . . . . . . . . . . . 4

1.3 Thesis Contributions . . . . . . . . . . . . . . . . . . 6

1.4 Thesis Structure . . . . . . . . . . . . . . . . . 7

2 Related Work $\quad 8$

2.1 Introduction to DSRC Standard . . . . . . . . . . . . . . . . 8

2.2 IEEE 1609.4 Standard Overview . . . . . . . . . . . . . . . . . . . . 11

2.3 Analytical Models of DSRC Multi-Channel Operation . . . . . . . . . . . 13

2.4 Algorithms Improving DSRC Performance and Reliability . . . . . . . . . . 14

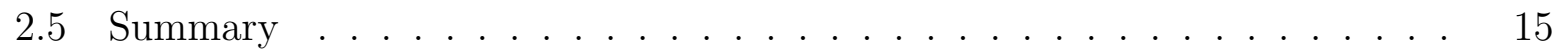

3 Analytical Models of 1609.4 Broadcasting and Unicasting 16

3.1 Assumptions . . . . . . . . . . . . . . . . . . . . 17

3.2 Markov Chain Model for 802.11p Broadcast in CCH . . . . . . . . . . . . . 18

3.2.1 Stationary probability . . . . . . . . . . . . . . 19

3.2.2 Blocking probability . . . . . . . . . . . . . . 19 
3.2 .3 Service Time . . . . . . . . . . . . . . . . . . . . 21

3.2.4 Modelling CCH and SCH Channel Switching . . . . . . . . . . 21

3.2 .5 Numerical Estimation $\ldots \ldots \ldots \ldots$

3.2 .6 Packet Loss Ratio . . . . . . . . . . . . . . . . . . . . . . 24

3.2 .7 Synchronized Collision . . . . . . . . . . . . . . . 25

3.3 Markov Chain Model for WAVE 1609.4 Unicast in SCHs _ . . . . . . 28

3.3 .1 System Throughput. . . . . . . . . . . . . . . . . . 30

3.4 Tradeoff in the models . . . . . . . . . . . . . . . . . . . . . 31

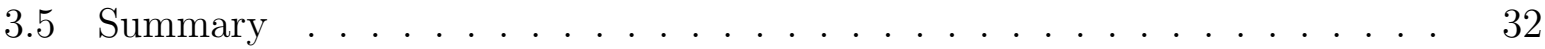

4 Multi-Channel Coordination Algorithm 33

4.1 The Multi-Channel Coordination Algorithm . . . . . . . . . . . . . 34

4.2 Algorithm Convergence and Convergence Rate . . . . . . . . . . . . . 38

4.3 Distributed consensus for estimating traffic density f . . . . . . . . 38

4.4 Summary . . . . . . . . . . . . . . . . . . . . . 39

5 Model Validation and Performance Analysis 40

5.1 Simulation Platform Introduction $\ldots \ldots \ldots$

5.2 Simulation $\operatorname{Setup~\ldots ...~.~.~.~.~.~.~.~.~.~.~.~.~.~} 41$

5.3 Model Validation . . . . . . . . . . . . . . . . . . . . . . . . . . . . . 42

5.4 Performance Evaluation $\ldots \ldots \ldots \ldots 4 . \ldots \ldots$

5.4.1 Impacts of Main Parameters on the Performance . . . . . . . . . 44

5.4 .2 Performance of the proposed algorithm $\ldots \ldots \ldots \ldots \ldots$

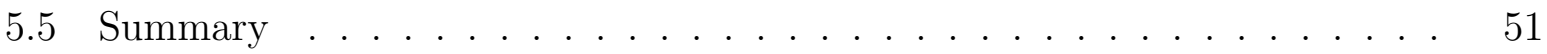

6 Conclusions and Future Directions $\quad 52$ 


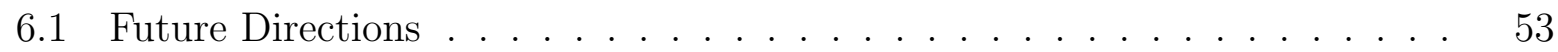

References 


\section{List of Figures}

1.1 General Motors DSRC system [22] . . . . . . . . . . . . . . . 2

1.2 DSRC vehicle-to-vehicle communication $[12] \ldots \ldots$. . . . . . . . . 2

1.3 USDOT recently sets up a Test Bed testing DSRC OBUs and RSUs in Michigan state $[43] \ldots \ldots \ldots \ldots \ldots$

$1.4 \mathrm{~V} 2 \mathrm{~V}$ and V2I highway scenario .................. . . . 5

2.1 DSRC layered architecture in the United States . . . . . . . . . . . 11

2.2 Four channel access options: (1) Continuous Access (2) Alternating Access (3) Immediate Access (4) Extended Access [2] . . . . . . . . . . . . . . . . 12

3.1 1-D Markov Chain for Control Channel improved over the EDCA model in paper $[52] \ldots \ldots \ldots \ldots \ldots \ldots$

3.2 1-D VANET Model [52] . . . . . . . . . . . . . . . . . . . . 24

3.3 Synchronized collision observed in NS-2 simulation. . . . . . . . . . . . 26

3.4 Synchronized collision at start of a channel interval . . . . . . . . . . . 27

3.5 2-D Markov Chain for Unicasting Service Channel improved over EDCA model in paper $[52] \ldots \ldots \ldots . \ldots \ldots$

4.1 Iterative Control System . . . . . . . . . . . . . . . . . 34 
5.1 PLR of CCH channel with BPSK and QPSK modulation . . . . . . . . 43

5.2 Throughput in SCHs for different packet sizes . . . . . . . . . . . . . 44

5.3 Percentage of packets sent during each time slice (Traffic density $=0.4$ vhls $/ \mathrm{m}$ ) 45

5.4 Impact of packet size and traffic density on Packet Loss Ratio in SCHs . . 46

5.5 Impact of traffic density on DSRC reliability . . . . . . . . . . . . . 47

5.6 Impact of $\mathrm{CCH}$ interval on $\mathrm{DSRC}$ reliability . . . . . . . . . . . . . 47

5.7 Impact of traffic density and $\mathrm{CCH}$ interval (CCHI) on Saturated Throughput

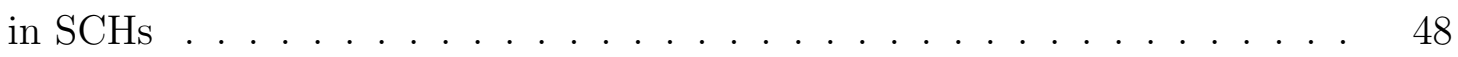

5.8 Optimal CCH interval length . . . . . . . . . . . . . . . . . 49

5.9 Packet Loss Ratio in $\mathrm{CCH}$ under the desired interval . . . . . . . . . . . 50

5.10 Throughput in SCHs under the desired interval . . . . . . . . . . . . 50

6.1 DSRC on-board unit can also communicate with smart-phone equipped pedestrians $[32] \ldots \ldots \ldots \ldots \ldots \ldots$

6.2 DSRC enabled smart-phone $[32] \ldots \ldots \ldots$. . . . . . . . . . . 54 


\section{List of Tables}

2.1 DSRC Standard in Europe and U.S. . . . . . . . . . . . . . . . 9

2.2 Safety Applications . . . . . . . . . . . . . . . . . . 10

2.3 Non-Safety Applications . . . . . . . . . . . . . . . . . . . . . 10

2.4 Comparison of Wireless Technologies _... . . . . . . . . . . . . 10

5.1 Simulation Configuration . . . . . . . . . . . . . . . . . 42 


\section{List of Acronyms}

16-QAM 16-point Quadrature Amplitude Modulation

64-QAM 64-point Quadrature Amplitude Modulation

AIFS Arbitration Inter-Frame Space

BPSK Binary Phase Shift Keying

$\mathrm{CCH} \quad$ Control Channel

CCHI Control Channel Interval

CSMA/CA Carrier Sense Multiple Access with Collision Avoidance

CTS Clear To Send

DIFS DCF Interframe Space

DSRC Dedicated Short Range Communications

DCF Distributed Coordination Function

EDCA Enhanced Distributed Channel Access

ETC Electronic Toll Collection

FCC Federal Communications Commission

GPS Global Positioning System

IETF Internet Engineering Task Force

IP Internet Protocol

MAC Medium Access Control 


\begin{tabular}{|c|c|}
\hline $\mathrm{OBU}$ & On-Board Unit \\
\hline OFDM & Orthogonal Frequency-Division Multiplexing \\
\hline PHY & Physical (Layer) \\
\hline PLR & Packet Loss Ratio \\
\hline PDR & Packet Delivery Ratio \\
\hline QPSK & Quadrature Phase Shift Keying \\
\hline RSU & Road Side Unit \\
\hline RTS & Request To Send \\
\hline $\mathrm{SCH}$ & Service Channel \\
\hline SCHI & Service Channel Interval \\
\hline SIFS & Short Interframe Space \\
\hline TTL & Time To Live \\
\hline UTC & Coordinated Universal Time \\
\hline V2I & Vehicle-to-Infrastructure \\
\hline $\mathrm{V} 2 \mathrm{~V}$ & Vehicle-to-Vehicle \\
\hline $\mathrm{V} 2 \mathrm{P}$ & Vehicle-to-Pedestrian \\
\hline VSC & Vehicle Safety Communication Consortium \\
\hline VANET & Vehicular Ad-Hoc Network \\
\hline WAVE & Wireless Access in Vehicular Environment \\
\hline WBSS & WAVE Basic Service Set \\
\hline WiFi & IEEE 802.11 Wireless LAN Standards \\
\hline WSA & WAVE Service Announcement \\
\hline WSM & WAVE Short Message \\
\hline WSMP & WAVE Short-Message Protocol \\
\hline
\end{tabular}




\section{Chapter 1}

\section{Introduction}

\subsection{Overview}

Traffic accidents and highway congestion are ongoing issues across the world. Unless special actions are taken, road injuries are foreseen to become the fifth leading cause of death by 2030. Globally, road crashes cost USD $\$ 518$ billion each year, which composes $1 \%-2 \%$ of the annual GDP per country. To tackle this issue, the United States (U.S.) Department of Transportation (DoT), together with industrial companies such as General Motors (GM) and Toyota, are focusing on developing the Dedicated Short-Range Communication (DSRC) standard to facilitate collision prevention applications including lane-changing assistance, road caution hazard notification, etc. [30]. Figure 1.1 shows a primitive demo of a DSRC on board unit (OBU) by GM. In order to enable vehicle-to-vehicle (V2V) and vehicle-toinfrastructure (V2I) communication, intelligent radio devices as radar, lidar, camera are currently in development. The lower level standards, i.e., physical layer protocol and MAC layer protocol, of DSRC have been established and approved by the Federal Communication Commission (FCC) and are at present being revised by IEEE 802.11p task group [20]. The 
latest amendment known as IEEE 1609.4 heralds that the era for DSRC is fast approaching.

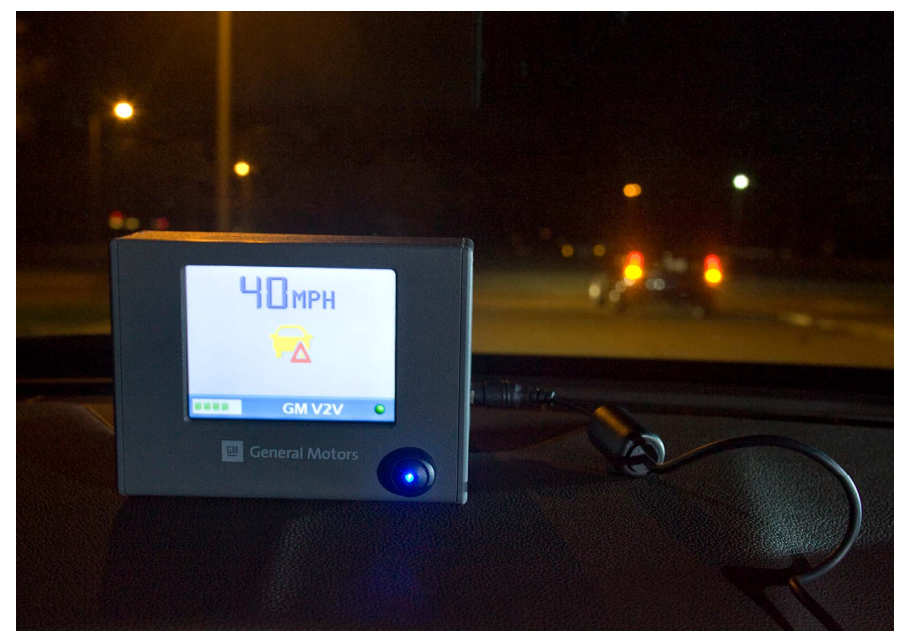

Fig. 1.1 General Motors DSRC system [22]

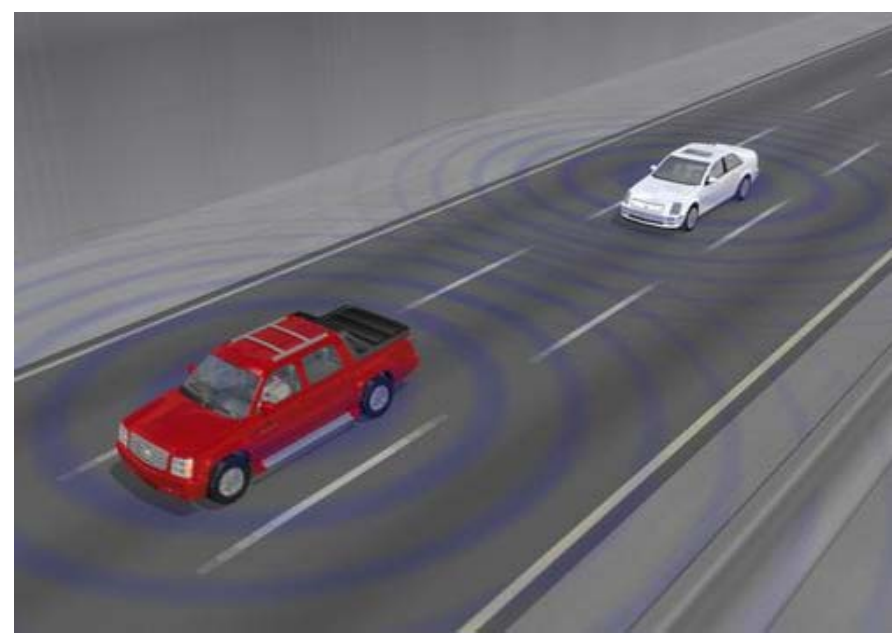

Fig. 1.2 DSRC vehicle-to-vehicle communication [12]

Figure 1.2 shows one scenario of $\mathrm{V} 2 \mathrm{~V}$ communication. Two vehicles exchange safety and position information via on-board units (OBUs). If a crash seems imminent, e.g. when inter-vehicle distance is too short, the cars will warn their drivers. Normally, the communication range can be as far as $450 \mathrm{~m}$, which is adequate to detect nearby traffic conditions. Figure 1.3 is an initial deployment of DSRC V2I system by USDOT in Michigan 
state [43]. The Test Bed is located on 75 miles of highway and arterial roadway located in Oakland County, Michigan. The Test Bed is equipped with 52 Dedicated Short Range Communications (DSRC) road side units (RSU) units. The DOT places RSUs to make radio ranges of RSUs overlap each other in order to see how vehicles and the system interact with multiple RSUs.

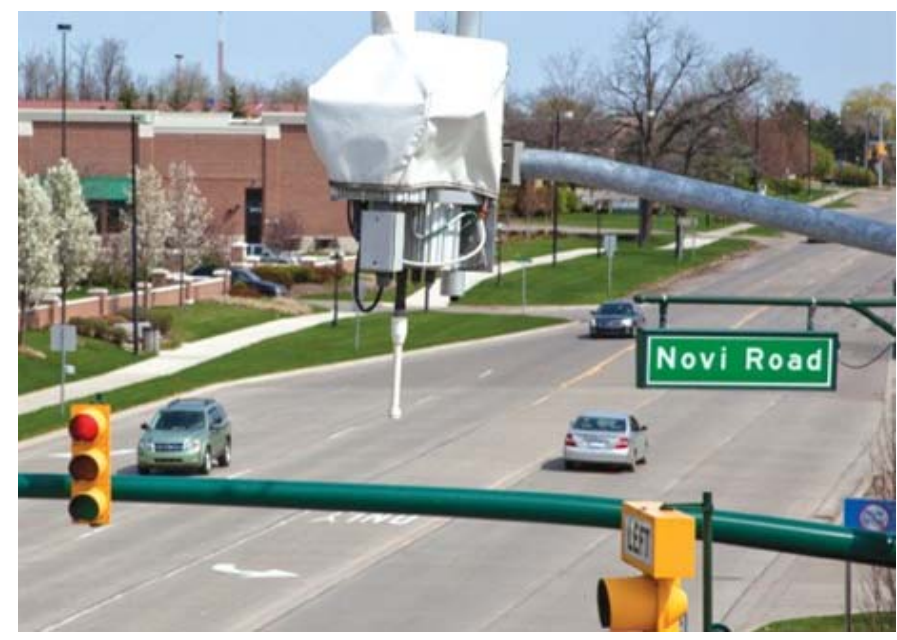

Fig. 1.3 USDOT recently sets up a Test Bed testing DSRC OBUs and RSUs in Michigan state [43]

Though the primary goal of DSRC is to ensure safe driving, the standard also supports a variety of non-safety related applications from electronic toll collection (ETC), drive-thru to multimedia downloading. The support of non-safety related communications offers a large pool of commercial opportunities which make DSRC devices more cost-effective. In this case, automobile manufacturers are motivated to equip their new vehicles with multiservice enabled DSRC radios. The potential profit of DSRC protocol encourages a greater market penetration.

Wireless Access in Vehicular Environments (WAVE) 1609.4 [2], an extension of DSRC, defines the multi-channel operation. The purpose of this extension is to ensure that multiradio devices can communicate with each other on the right channel. There are seven 
channels defined in this extension. One channel known as Control Channel $(\mathrm{CCH})$ is allocated to transmit safety related messages as well as WAVE control messages. The other six channels named Service Channels (SCHs) are reserved for non-safety communication. The extension further defines a division of time intervals into alternating $\mathrm{CCH}$ intervals (CCHI) and SCH intervals (SCHI) for single-radio devices [10]. A guard interval is also added in front of each interval to account for time inaccuracies and operational delay. Therefore, devices switch back and forth between $\mathrm{CCH}$ and $\mathrm{SCH}$ on a Time Division Multiple Access (TDMA) basis. Recently, voices from industry [30] suggest that safety messages should be transmitted on a dedicated channel without time division. Vehicles that are interested in both safety and non-safety data on dedicated channels have to be equipped with at least two radios, one consistently tuned to the safety channel and another involved in WAVE 1609.4 switching. At the initial deployment phase of DSRC, both types of devices will likely appear as there is a tradeoff between radio cost and service quality. Hence, algorithms designed to enhance the performance of the protocol should be comfortable to cope with either scenario.

\subsection{Problem Definition}

While supporting both safety and non-safety applications, channel switching also brings novel characteristics to 802.11 p. First of all, it is mandatory that safety messages are generated in CCH interval to prevent potential Synchronized Collisions [13]. Second, transmission medium for a channel has to be declared as busy when the channel is not operating. Thus, no packet from this channel can be transmitted during that time. Third, if the backoff process has not yet completed at the end of the channel interval, it should be frozen and must be restarted when the next channel interval begins. 
Furthermore, there are four channel access options defined in the 1609.4, namely continuous access, alternating access, extended access and immediate access, as Figure 2.2 demonstrates. With continuous access, a radio is always tuned to a $\mathrm{CCH}$ or $\mathrm{SCH}$. Alternating access is the default channel access approach which equally divides the synchronization interval between $\mathrm{CCH}$ and $\mathrm{SCH}$. The drawback of this option is significant as the channel capacity is halved. Safety messages can not be transmitted during SCH interval (SCHI) and non-safety services are not available during $\mathrm{CCH}$ interval (CCHI). Additionally, as backoff process in one channel has to be frozen if we do not operate on that channel, packets in the queue associated with that channel will suffer extra delay [37]. Extended Access allows vehicles to stay on SCHs for successive synchronization intervals without switching to CCH. Immediate access allows radios to perform channel switching at any time. Channel capacity could be improved in this approach. However, it still remains an open issue on how to determine the switching point for better reliability and performance. In this work, we focus on how to adaptively adjust the channel interval to balance $\mathrm{CCH}$ packet loss and SCH throughput.

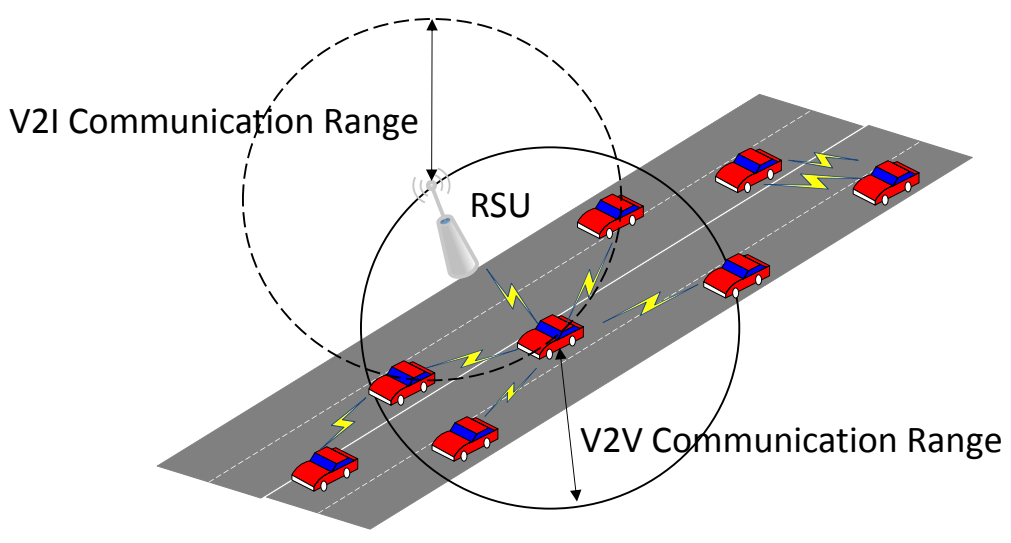

Fig. 1.4 V2V and V2I highway scenario

Current research mainly focuses on modelling continuous access based 802.11 protocol 
while there is little work concerning channel switching. Moreover, the default channel access approach, alternating access, adopts a fixed channel interval, which can not cope with dynamic scenarios of safety and non-safety applications. In a highway scenario, the network topology is unstable due to the high mobility of vehicles. Fixed channel switch can not not maximize the chance for successful data exchange in V2V or V2I communication, which is a critical flaw hindering DSRC commercialization.

In this thesis, we assume that Road Side Unit (RSU) will be present to provide infotainment applications to vehicles. Hence, we have to guarantee the successful infotainment transmission within small contact time between RSU and nodes. Moreover, once a DSRC network is deployed, it is assumed that main parameters remain constant except traffic densities. The problem that how to divide the operating interval to balance PLR in $\mathrm{CCH}$ and throughput in SCHs is what we focus. We propose a multi-channel coordination algorithm based on Markov Chain (MC) to tackle this problem. We take traffic densities as

a feedback signal to our algorithm. Our models capture the relationship between packet loss ratio and traffic density. When the density is high, the $\mathrm{CCH}$ channel interval will be increased to provide more bandwidth for safety messages. If the density is small, the $\mathrm{CCH}$ interval will be reduced ato increase the throughput of non-safety services in SCHs. As a result, These operations allow the CCHI to adapt to dynamic scenarios.

\subsection{Thesis Contributions}

There are three main contributions of this thesis:

1. We improve previous models on 802.11 EDCA protocol [9] [52] [51], to capture the features of channel switch. The models explore the relationship among traffic density, $\mathrm{CCH}$ packet loss ratio, $\mathrm{SCH}$ throughput, and the duration of each kind of intervals. 
2. We propose an iterative control based multi-channel coordination algorithm, to dynamically adapt channel intervals according to the instant traffic density.

3. We conduct extensive simulations and show that the proposed algorithm almost doubles throughput in SCHs in low traffic density scenario and reduces packet loss ratio up to $30 \%$ in high traffic density scenario.

It is worth noting that the proposed algorithm works with both single radio and multiradio devices. Therefore there is no hardware dependency for our algorithm. This feature is quite beneficial for automobile manufacturers in the early stage of DSRC deployment. To the best of our knowledge, this is the first work that models the reliability of WAVE 1609.4 protocol and dynamically adjusts channel interval without modifying the existing standard.

\subsection{Thesis Structure}

The rest of this thesis is organized as follows. The related work is introduced in Chapter II; Performance metrics and Markov Chain models for WAVE 1609.4 multi-channel operation in both broadcast and unicast modes are presented in Chapter III; The multi-channel coordination algorithm is discussed in Chapter IV; Chapter V conducts extensive simulations to validate the accuracy of the model and effectiveness of the proposed algorithm; Chapter VI closes this thesis with concluding remarks. 


\section{Chapter 2}

\section{Related Work}

In this chapter, we first briefly introduce the DSRC protocol and IEEE 1609.4 amendment. Then we review related research papers that focus on enhancing the performance and reliability of the DSRC protocol.

\subsection{Introduction to DSRC Standard}

The Dedicated Short-Range Communication (DSRC) protocol is a short-range wireless communication protocol that offers fast and reliable data transfer in vehicular ad-hoc network (VANET) [6]. The main characteristics of VANET are the high topological dynamics and the infrastructure absence such as access point or base stations existing in the Wi-Fi, WiMax. Moreover, it also introduces new challenges in comparison to traditional wireless networks. First, the high mobility in VANET requires a protocol that can can support fast communications among vehicles. Second, interference from other hot spots is detrimental to real time vehicle communication. DSRC is well designed to solve these two problems: it provides immediate establishment of communication and it is allocated in a dedicated 
frequency band [19]. Governments have shown great interests in this emerging technology. Table 2.1 summarizes the differences of this protocol in European Union and United States and Table 2.4 compares DSRC with traditional Wi-Fi technology.

Table 2.1 DSRC Standard in Europe and U.S.

\begin{tabular}{|c|c|c|}
\hline & European Union & United States \\
\hline International Standard & $\begin{array}{c}\text { ITU-R M.1453-2 (Layer-1) } \\
\text { ISO 15628 (Layer-2,7) }\end{array}$ & ISO 21215 (CALM M5) \\
\hline Radio Frequency & $5.8 \mathrm{GHz}$ & $5.9 \mathrm{GHz}$ \\
\hline Frequency Range & $5.85-5.925 \mathrm{GHz}$ & $5.855-5.905 \mathrm{GHz}$ \\
\hline Downlink rate & $500 \mathrm{Kbps}$ & $2-27 \mathrm{Mbps}$ \\
\hline Uplink rate & $250 \mathrm{Kbps}$ & $2-27 \mathrm{Mbps}$ \\
\hline Communication rage & up to $5 \mathrm{~m}$ & up to $1000 \mathrm{~m}$ \\
\hline
\end{tabular}

The spectrum of DSRC is divided into seven channels, namely, one control channel $(\mathrm{CCH})$ and six service channels (SCHs), each of which has $10 \mathrm{MHz}$ bandwidth. In a single radio device, it is supposed to support both kinds of channels. DSRC shall schedule it on a TDMA basis. In dual-radio devices, one radio is always tuned to $\mathrm{CCH}$ and another radio is always tuned to SCH. The physical layer of DSRC is similar to that of IEEE 802.11a, which utilizes the Orthogonal Frequency Division Multiplexing (OFDM) technique. The MAC layer utilizes IEEE 802.11p, which is based on Carrier Sense Multiple Access with Collision Avoidance (CSMA/CA). For safety applications, broadcast packets are transmitted via $\mathrm{CCH}$. For non-safety applications, as different user has different demands, it is supposed to use unicast to transmit via SCH. Moreover, to overcome hidden terminals, Request-To-Send (RTS)/Clear-To-Send (CTS) packets are also included in the standard.

There are a number of important applications of DSRC. We summarize safety applications in Table 2.2 and non-safety applications in Table 2.3 .

Figure 2.1 presents a layered DSRC architecture. It also follows the seven layer architecture in the OSI model. Besides, new amendments called IEEE 1609 family [2] are 
Table 2.2 Safety Applications

\begin{tabular}{|l|l|l|}
\hline $\begin{array}{l}\text { Intersection Collision } \\
\text { Avoidance }\end{array}$ & Public Safety & Sign Extension \\
\hline Traffic Signal Violation Warning & $\begin{array}{l}\text { Approaching Emergency Vehicle } \\
\text { Warning }\end{array}$ & In-Vehicle Signage \\
\hline Blind Merge Warning & $\begin{array}{l}\text { Emergency Vehicle Signal Pre- } \\
\text { emption }\end{array}$ & Curve Speed Warning \\
\hline Left Turn Assistant & SOS Services & Low Parking Structure Warning \\
\hline Intersection Collision Warning & Post-Crash Warning & Wrong Way DriverWarning \\
\hline
\end{tabular}

Table 2.3 Non-Safety Applications

\begin{tabular}{|l|l|l|}
\hline Traffic Management & $\begin{array}{l}\text { Information from OtherVe- } \\
\text { hicles }\end{array}$ & Tolling \\
\hline Intelligent On-Ramp Metering & Cooperative Glare Reduction & Drive-thru payment \\
\hline Intelligent Traffic Flow Control & Instant Messaging & Parking lot payment \\
\hline Traffic information & Adaptive Headlamp Aiming & Free-Flow Tolling \\
\hline $\begin{array}{l}\text { Infrastructure-based traffic } \\
\text { management probes }\end{array}$ & $\begin{array}{l}\text { Adaptive Drivetrain Manage- } \\
\text { ment }\end{array}$ & \\
\hline
\end{tabular}

proposed to enhance DSRC's performance. IEEE 1609.2 addresses security and privacy issues. IEEE 1609.3 defines networking layers. IEEE 1609.4 is a sub MAC layer that rules multi-channel operation and is the main focus of this thesis.

Table 2.4 Comparison of Wireless Technologies

\begin{tabular}{|c|c|c|}
\hline & DSRC & Wi-Fi \\
\hline Frequency band & $5.9 \mathrm{GHz}$ & $5 / 2.4 \mathrm{GHz}$ \\
\hline Channel Bandwidth & $10 \mathrm{MHz}$ & $20 \mathrm{MHz}$ \\
\hline Supported Data Rate & $3,4,5,6,9,12,18,24$ & $6,9,12,18,24,36$ \\
\hline Modulation & BPSK, QPSK, $16 \mathrm{QAM}, 64 \mathrm{QAM}$ & Same as DSRC \\
\hline Channel Coding & $1 / 2,2 / 3,3 / 4$ & Same as DSRC \\
\hline FFT/IFFT Interval & $6.4 \mu \mathrm{s}$ & $3.2 \mu \mathrm{s}$ \\
\hline Subcarrier spacing & $0.15625 \mathrm{MHz}$ & $0.3125 \mathrm{MHz}$ \\
\hline OFDM Symbol & $8 \mu \mathrm{s}$ & $4 \mu \mathrm{s}$ \\
\hline
\end{tabular}

DSRC is preferred over Wi-Fi because the proliferation of Wi-Fi hand-held and handsfree devices that occupy the $2.4 \mathrm{GHz}$ and $5 \mathrm{GHz}$ bands, along with the projected increase in Wi-Fi hot spots and wireless mesh extensions can cause intolerable and uncontrollable levels of interference for vehicular safety applications [47]. DSRC is developed primarily 


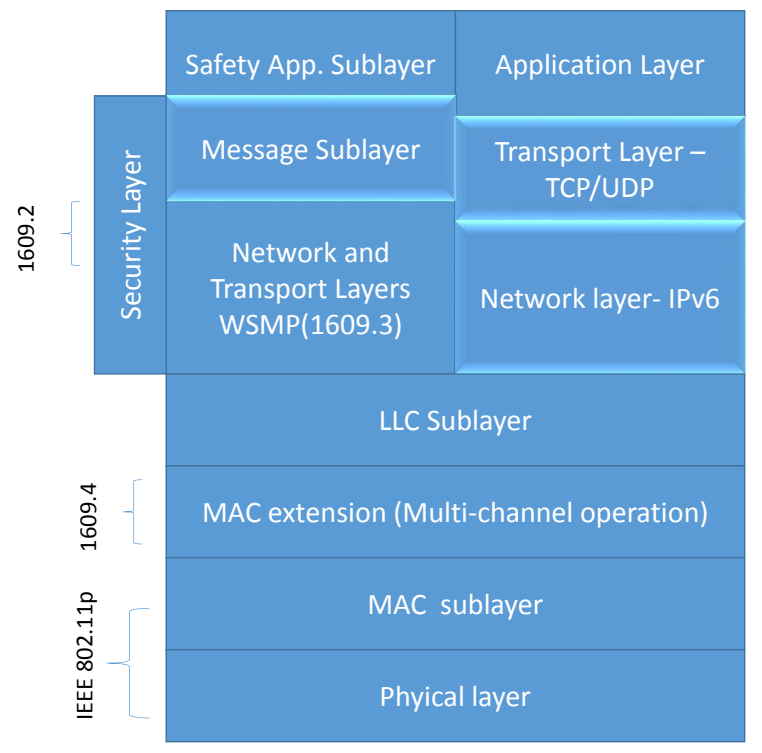

Fig. 2.1 DSRC layered architecture in the United States

for safety applications and addresses the interference issues. Moreover, DSRC is the most important short-range wireless technology today in vehicular networks that provides fast network handover, low latency, high reliability and good security.

\subsection{IEEE 1609.4 Standard Overview}

The IEEE 1609.4 is a MAC extension that supports multi-channel operations in DSRC. It allows devices to tune to the same channel at the same time. The "rendezvous" channel known as Control Channel is allocated to transmit safety related messages as well as WAVE control messages. Six channels named Service Channel in DSRC spectrum are reserved for non-safety communication. The extension further defines a division of operating intervals into $\mathrm{CCH}$ intervals and SCH intervals. A guard interval is also added in front of each interval to account for time inaccuracies and operational delay. Effectively, devices switch back and forth between $\mathrm{CCH}$ and $\mathrm{SCH}$ on a TDMA basis. 
Recently, voices from industry [30] suggest that safety messages should be transmitted on a dedicated channel without involving time division. For vehicles that are interested in both safety and non-safety data, this change entails equipping vehicles with at least two radios, one consistently tuned to $\mathrm{CCH}$ and another involving in WAVE 1609.4 switching. At the initial deployment phase of DSRC, both types of devices will likely appear as there is a tradeoff between radio cost and service quality. Hence, algorithms designed for enhancing the performance of the protocol should be comfortable coping with either scenario.

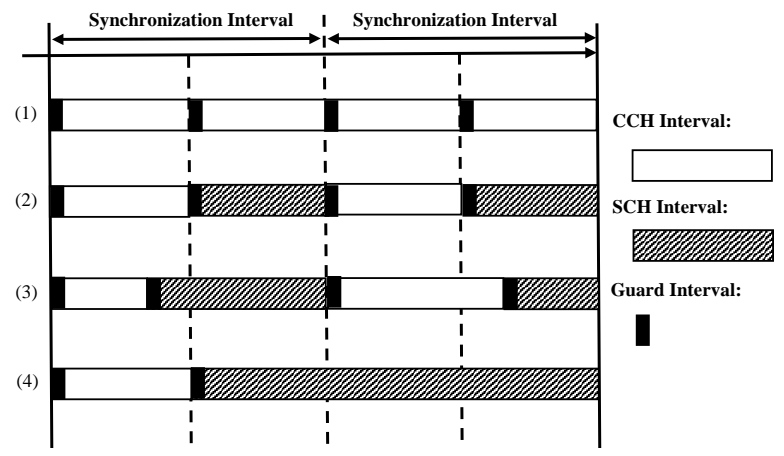

Fig. 2.2 Four channel access options: (1) Continuous Access (2) Alternating Access (3) Immediate Access (4) Extended Access [2]

In terms of channel coordination, there are four channel access options defined in the 1609.4, namely continuous access, alternating access, immediate access and extended access, as figure 2.2 demonstrates. According to continuous access, a radio is always tuned to a $\mathrm{CCH}$ or $\mathrm{SCH}$ without any switching performed. Alternating access is the default channel access approach which equally divides the whole synchronization interval (100 ms) for $\mathrm{CCH}$ and $\mathrm{SCH}$. Immediate access allows radios to perform channel switching at any time. Channel capacity could be saved in this approach. Extended access allows vehicles to stay on $\mathrm{SCH}$ for successive synchronization intervals without switching back to $\mathrm{CCH}$. 


\subsection{Analytical Models of DSRC Multi-Channel Operation}

Motivated by problems brought by wireless network dynamics, a number of works address the issue of modelling 802.11 MAC protocol in general such as [9] and in particular for vehicular networks such as [52] [11]. Bianchi [9] first models 802.11 DCF using Markov Chain. Wang et al. [48] study the performance and throughput of a IEEE 802.11p like MAC protocol based on Bianchi's model. While time for transmitting safety related messages in $\mathrm{CCH}$ is calculated, the safety message broadcast is not analysed and dynamic switch between $\mathrm{CCH}$ and $\mathrm{SCH}$ is excluded in the model. Yao et al. [52] further propose a model for 802.11p EDCA protocol. In this paper, priority of different traffic classes and virtual collisions are considered. However, these models only work with the single channel operation. Campolo et al. [11] leverage the Bernoulli process to model prioritized broadcasting in multi-channel vehicular networks. In their paper, the time to live (TTL) of each safety-related message is assumed to be $100 \mathrm{~ms}$, implying that packets failing to be sent in the current synchronization cycle shall be discarded. Nonetheless, this assumption does not reveal the general scenario defined by the standard where packets can be stored in queue and transmitted in next synchronization interval. Moreover, hidden terminals are not considered and the unicasting in SCHs is not modelled. Misić et al. [41] analyse the delay of 802.11p network with single channel devices. Several traffic combinations are accounted for both $\mathrm{CCH}$ and $\mathrm{SCH}$. The impact of the interruption of the backoff process by inactive channel time is quantitatively analysed. However, synchronized collision is not captured in the model. This model also grounds the selection of optimal $\mathrm{CCH}$ interval according to the policy of the network operator.

Though 802.11 protocols have been extensively explored, a model that fully characterizes the DSRC multi-channel network is still left blank. To this end, we improve previous 
DSRC models in multi-channel context. The analytical models embrace following features: (1) both broadcasting safety-related messages in $\mathrm{CCH}$ and unicasting non-safety-related services in SCHs are included in the model; (2) impact brought by channel switching is explicitly revealed; (3) metrics like PLR and throughput are adopted to evaluate the system's performance; (4) both saturated and non-saturated network conditions are covered by the model.

\subsection{Algorithms Improving DSRC Performance and Reliability}

Existing work shows that the default alternating channel access option in multi-channel environment neither provides sufficient reliability for safety-related message delivery [13] [53] nor satisfactory throughput for infotainment services delivery [49] [44]. Techniques to solve this problem have been broadly studied. Kenney et al. [26] propose a new design of WAVE 1609.4 protocol, which includes an extra bit in the packet header to show the nodes' intention to keep staying on $\mathrm{CCH}$ or to operate on multi-channels. This work enables the co-existence between single radio nodes and multi-radio nodes and guarantees the reliability of safety related messages. D. Jiang et al. [17] introduce a "peercast" protocol to enable vehicles to asynchronously switch channels. In this paper, vehicles are not required to receive all safety messages to guarantee reliability. Thus, they can selectively listen to $\mathrm{CCH}$, hence, spend more time in $\mathrm{SCH}$. Tony et al. [36] propose a multi-channel MAC protocol that leverages a Road Side Unit (RSU) to coordinate channels and differentiate distances to service coverage area. Kai et al. [31] and Han et al. [14] also shed insights on how to design a multi-channel MAC protocol to minimize collision. Wang et al. [48] adjust the $\mathrm{CCH}$ time interval by quantitatively calculating the time required to send safety messages, hence SCH throughput may change accordingly. Nonetheless, this is a static 
approach that does not explicitly guarantee reliability. Moreover, time synchronization problem is not appropriately handled. To summarize, there is no analytical model that includes both basic features of IEEE 802.11p and WAVE 1609.4 multi-channel protocol. In addition, there is also no dynamic solution that adjusts the interval length to meet requirements of reliability and throughput in multi-channel environment.

In order to tackle these challenges, we propose a multi-channel coordination algorithm that is based on the feedback signal of current reliability, which is calculated based on the proposed models. For every interval update period, if the reliability is detected as "low", $\mathrm{CCH}$ intervals will be enlarged to alleviate synchronized collision. Conversely, if the reliability is higher than a pre-defined threshold, $\mathrm{CCH}$ intervals will be reduced to give more time for infotainment services transmission in SCHs. Our algorithm properly handles time synchronization between vehicles and works well with the existing standard.

\subsection{Summary}

In this chapter, we briefly introduce basics of DSRC protocol as well as its newest amendment IEEE 1609.4. We also review existing work on analytical models of IEEE 802.11 MAC protocol and algorithms to improve the performance and reliability of DSRC. Finally, we show there are still challenges in providing satisfactory reliability and throughput in multichannel operation, to which end we propose new analytical models and a multi-channel coordination algorithm. 


\section{Chapter 3}

\section{Analytical Models of 1609.4 Broadcasting and Unicasting}

Performance and reliability analysis of the IEEE 802.11p MAC layer is an important and challenging problem that attracts considerable amount of attention from researchers. However, many existing works only address basic features of IEEE 802.11p. The newly proposed standard IEEE 1609.4 which supports multi-channel operations has not been fully explored and the synchronized collision introduced by multi-channel operations has not yet been analysed. In this chapter, we assume safety messages are broadcasted in $\mathrm{CCH}$ and non-safety related messages are unicasted in SCH. This assumption is based on the fact that safety messages are used to alert all vehicles nearby and non-safety related messages such as MP3 downloading are specific to a vehicle. We elaborate analytical models for both scenarios. Besides the fundamental EDCA MAC protocol, we also consider the 1609.4 MAC sub-layer extension. Metrics showing the performance and reliability of the system are also derived based on proposed analytical models. 


\subsection{Assumptions}

In this chapter, we focus on the reliability and performance analysis of the broadcasting in $\mathrm{CCH}$ and unicasting in SCHs. We consider each vehicle as a node in our model and several practical factors that impact the performance of DSRC radios. In our models, we assume IEEE 802.11p and IEEE 1609.4 work under following scenarios.

1. The instant positions of vehicles follow a Poisson distribution on a single lane. This assumption has been verified with real-world data in [21]. Thus, Given the traffic density $\beta$, the probability $P(i, l)$ of finding $i$ nodes in length $l$ is

$$
P(i, l)=\frac{(\beta l)^{i} e^{-\beta l}}{i !}
$$

2. Packet arrivals at each node follow a Poisson process with arrival rate $\lambda$. And the packet service time at each node follows a general distribution. Therefore, the buffer at each node can be modelled as a M/G/1 queue.

3. All nodes have the same transmission range, $R$. The mean number of nodes within transmission range of a tagged node is $N_{t r}=2 \beta R$.

4. All nodes have the same carrier sense range, $L_{c s}$, the mean number of nodes within the carrier sensing range of the tagged node is $N_{c s}=2 \beta L_{c s}$.

5. We assume that $\mathrm{CCH}$ and $\mathrm{SCH}$ adopt the same modulation technique to ensure that their transmission ranges are identical.

6. As is shown in Figure 3.2, the potential hidden terminal areas of the tagged node are $\left[L_{c s}, R+L_{c s}\right]$ and $\left[-R-L_{c s},-L_{c s}\right]$. We assume $L_{c s}$ equals to the interference range $L_{i n t}$, the average number of potential hidden nodes is $N_{p h}=4 \beta R$. 
7. We neglect the Doppler shift caused by the mobility of vehicles . According to [33], vehicles with a speed of $120 \mathrm{mi} / \mathrm{h}$ will only move $0.053 \mathrm{~m}$ during a packet transmission time (packet size is $200 \mathrm{~B}$ and data rate is $12 \mathrm{Mb} / \mathrm{s}$ ). It is orders of magnitude smaller than the lane length.

8. We assume that RTS/CTS scheme is turned off in our scenario to minimize communication overhead.

9. We consider all traffics are of the same access class.

\subsection{Markov Chain Model for 802.11p Broadcast in CCH}

This model improves previous models as it includes both channel switch and synchronized collision. The states of our Markov Chain model are a set of possible backoff counters, denoted as $\{b(t)\}$. The backoff counter down-counts the number of idle time slots that a node has to wait before transmission. If the counter reaches zero, it entails that the node is ready to transmit. Moreover, as there is no feedback acknowledgement from the receiver if the packet is successfully transmitted or not, the transmitter does not have any idea about the network condition. Hence, the contention window will be kept constant. We model this process as a 1-D Markov Process as Figure 3.1 illustrates. In Figure 3.1, IDLE is the state that there is no packets ready to be sent. The states $\left\{0, \ldots, W_{0}-1\right\}$ denote the value of the backoff counter. $p_{b}$ is the probability that the node senses other nodes occupying the channel. $p_{\text {cch }}$ is the probability that $\mathrm{CCH}$ is the current operating interval. $p_{\text {sch }}$ is the probability that $\mathrm{SCH}$ is the current operating interval. 


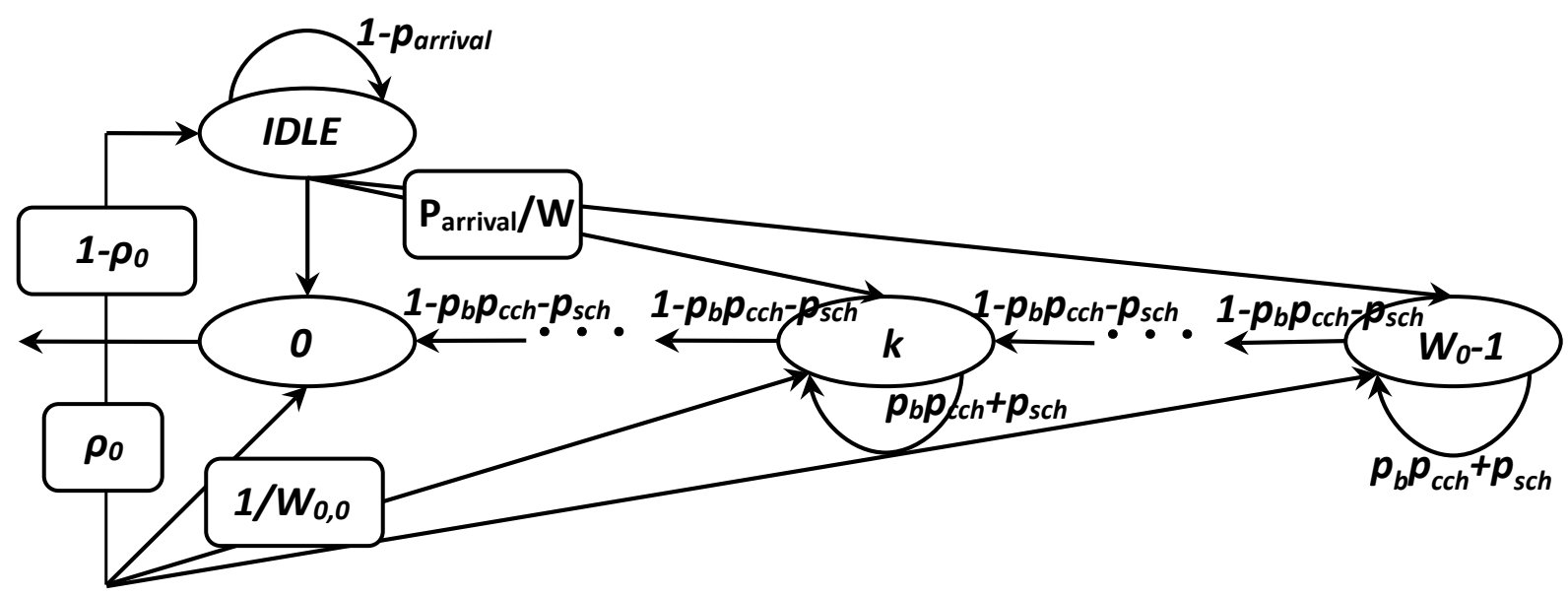

Fig. 3.1 1-D Markov Chain for Control Channel improved over the EDCA model in paper [52]

\subsubsection{Stationary probability}

We first would like to know the stationary probability of each states. Let $\pi_{k}$ be the stationary probability of state $\{b(t)=k\}$ and $\pi_{I D L E}$ be the stationary probability of state $I D L E$, i.e. when there is no packet running the backoff process. We have following balance equations between states:

$$
\begin{aligned}
& \pi_{I D L E}=\frac{1-\rho}{P_{\text {arrival }}} \pi_{0}, \\
& \pi_{k}=\frac{W_{0}-k}{(1-p) W_{0}} \pi_{0},
\end{aligned}
$$

where $\rho$ is the packet queue utilization in $\mathrm{CCH}$ and $\rho=\min \left(1, \frac{\lambda_{C C H}}{\mu_{C C H}}\right)$.

\subsubsection{Blocking probability}

Next, we derive the blocking probability in the MC in order to get an analytical solution. Since the backoff process will be blocked when the medium is sensed busy, which includes two cases, either the node detects there is a transmission on going in $\mathrm{CCH}$ or the operating 
interval is in SCHs. The overall blocking probability is

$$
p=\left(1-\frac{T_{C C H I}}{T_{S Y N C}}\right)+\frac{T_{C C H I}}{T_{S Y N C}} p_{b}
$$

where $p_{b}$ is the probability that a node finds an on going transmission in CCH, $T_{C C H I}$ is the $\mathrm{CCH}$ interval and $T_{S Y N C}$ is synchronization interval.

Since the packet arrival rate follows a Poisson distribution, the probability that a packet arrives in a generalized slot time is

$$
P_{\text {Arrival }}^{C C H}=\sum_{k=1}^{\infty} \frac{\left(\lambda_{C C H} \sigma_{C C H}^{\prime}\right)^{k}}{k !} e^{-\lambda_{C C H} \sigma_{C C H}^{\prime}}=1-e^{-\lambda_{C C H} \sigma_{C C H}^{\prime}}
$$

where an average slot time is

$$
\sigma_{C C H}^{\prime}=p_{b} T^{\prime}+\left(1-p_{b}\right) \sigma
$$

where $\sigma$ is a normal time slot and $T^{\prime}$ is the average time that the backoff counter will be fronzen when the channel is sensed busy, which is

$$
T^{\prime}=\frac{L_{H}+\mathbb{E}[P]}{R_{d}}+D I F S+\delta
$$

where $L_{H}$ is the packet header, $\mathbb{E}[P]$ is the packet length, $R_{d}$ denotes the data rate, $\delta$ represents the propagation delay and DIFS is the Distributed Inter-frame Spacing in the 802.11 protocol. Summing up all stationary probabilities in the Markov Chain, we have

$$
\pi_{I D L E}+\sum_{k=0}^{W_{0}-1} \pi_{k j}=1
$$


Thus, the stationary state probability $\pi_{0}$ is

$$
\begin{aligned}
\pi_{0} & =\left[\frac{W_{0}+1}{2(1-p)}+\frac{1-\rho}{p_{\text {arrival }}}\right]^{-1} \\
= & {\left[\frac{W_{0}+1}{2\left\{1-\left[1-\frac{T_{C C H I}}{T_{S Y N C}}+\frac{T_{C C H I}}{T_{S Y N C}}\left(1-e^{-\pi_{0}\left(N_{c s}-1\right)}\right)\right]\right\}}\right.} \\
& \left.+\frac{1-\rho}{1-e^{-\lambda_{C C H} \sigma}}\right]^{-1} .
\end{aligned}
$$

Also, as it models the broadcast mode, this is also probability, $\tau_{C C H}$, that any node transmits in an arbitrary time slot.

\subsubsection{Service Time}

It is critical to know the service time as we need it later to find the queue utilization. Arrival process is modelled as a Poisson process and we characterize the service time as a general distribution. Hence, each node works as a $\mathrm{M} / \mathrm{G} / 1$ queue. Denote $q_{i}$ as the steady state probability that the service time is $i$ time slots. Let $Q(z)$ be the Probability Generating Funtion $(P G F)$ of $q_{i}$ and it can be represented as,

$$
Q(z)=\sum_{i} q_{i} z^{i}
$$

Its first order derivative is the service time that we are looking for.

\subsubsection{Modelling $\mathrm{CCH}$ and SCH Channel Switching}

$\mathrm{CCH}$ and SCH channel switching is a new feature that we shall include in our model. Each synchronization interval is divided into a $\mathrm{CCH}$ interval and a SCH interval. There are separate backoff counters in each channel. How to integrate different channels into one model remains a problem. We understand that the backoff counter in each mobile node will 
be decremented by a slot once an idle channel is sensed for a DCF Interframe Space (DIFS). Conversely, it will wait for an extra period once the channel is sensed busy. Moreover, if the backoff starts near the end of CCHI, there is a high likelihood that it can not be completed within this interval. As a penalty, it has to wait for a period of SCH interval and guard interval. When the next cycle comes, it will be unblocked and continue the backoff process. As it is mentioned in Section III-A, the probability that a node waits for two consecutive cycles approaches to zero even when there is a congestion in the network [40]. Otherwise the the queuing delay will be too long to satisfy real-time requirement. Therefore, for a tagged transmitting node in broadcast communication, the time for the backoff counter decrementing by one can be modelled by the following $P G F$

$$
\begin{array}{r}
H_{\text {total }}(z)=\left(1-e^{-\lambda_{C C H}(C C H I-T)}\right)\left[\left(1-p_{b}\right) z+p_{b} z^{\frac{T}{\sigma}}\right] \\
+e^{-\lambda_{C C H}(C C H I-T)} z^{\frac{S Y N C-C C H I}{\sigma}}\left(\left(1-p_{b}\right) z+p_{b} z^{\frac{T}{\sigma}}\right) .
\end{array}
$$

Hence, the PGF of the service time is obtained from the product of the PGF of the backoff decrementation time and the transmission time, which is

$$
Q_{\text {total }}(z)=\frac{z^{\frac{T-D I F S}{\sigma}}}{W_{0}} \sum_{i=0}^{W_{0}-1}\left(H_{\text {total }}(z)\right)^{i}
$$

As a result, the first moment of the service time can be calculated from the above PGF which is in the following form

$$
T_{\text {total }}^{\text {ave }}=\sum_{i}^{\infty} q_{i}=\left.\frac{d Q_{\text {total }}(z)}{d z}\right|_{z=1}
$$


By definition, the service rate of the queue is achieved as

$$
\mu_{C C H}=\frac{1}{T_{\text {total }}^{a v e}} .
$$

\subsubsection{Numerical Estimation}

After building the above model, we can numerically quantify the service rate, service time and packet transmission probability in an arbitrary slot. To calculate the service rate, we have to know the queue utilization $\rho$. However, $\rho$ itself is a function of $\mu_{C C H}$. Thus, we need to apply the following iterative method to solve equations from (3.9) to (3.14).

Step 1 Initialize $\rho$ based on the estimation of the network condition, i.e. If the network is unsaturated, it is better to initialize the value with 0 .

Step 2 Solve the multivariate nonlinear equations which are composed by equation (3.9), (3.11), (3.12) and (3.14).

Step 3 Calculate the average service rate according to equation (3.14).

Step $4 \rho_{0 \text { new }}=\min \left(\lambda_{C C H} T_{s}, 1\right)$. If $\rho_{0 \text { new }}-\rho<\epsilon$, the iteration is completed, where $\epsilon$ is a pre-defined error bound. Otherwise, go to Step 2 with $\rho_{0 \text { new }}$

Comparing multi-channel operation with single channel operation in IEEE 802.11p, we can quantify the service time caused by channel switching as follows

$$
\Delta T=T_{\text {total }}^{\text {ave }}-T_{c}^{\text {ave }} .
$$

where $T_{\text {ave }}^{c}$ is the mean service time for $802.11 \mathrm{p}$ that operates in continuous access and defined in [35] and $T_{\text {ave }}^{c}=\left.\frac{d Q(z)}{d z}\right|_{z=1}$. 


\subsubsection{Packet Loss Ratio}

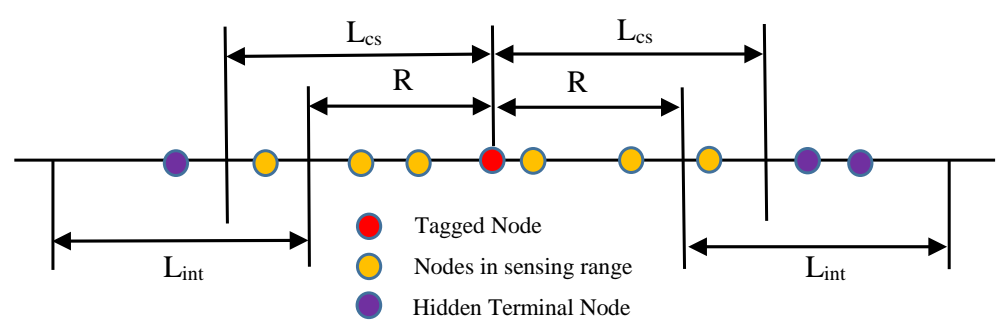

Fig. 3.2 1-D VANET Model [52]

Packet Loss Ratio (PLR) [7] is an indispensable metric quantifying reliability. It can be calculated as a complement of Packet Delivery Ratio (PDR). PLR is defined as the probability of failing to receive a packet at the receiver after this packet is transmitted at the sender. To calculate PLR in a vehicular scenario, we consider a 1-D VANET scenario as shown in Figure 3.2. Both hidden terminals and concurrent transmissions of nodes within carrier-sensing range of the tagged node have negative impact on PLR. Following the approach in [35], we are able to obtain the analytical form of PDR and then get its complement to have PLR. Due to space limitation, we hereby directly present the final results. Please refer to [35] for more detail.

Define $\Delta L=L_{c s}-L_{i n t}, C=\beta T_{\text {vuln }} \tau_{C C H} / t_{s}$, where $\beta$ is the traffic density, $T_{\text {vuln }}$ is the vulnerable period that the transmission suffers from hidden terminal problem and $t_{s}$ is the average time the transmission takes. From previous sections, we have calculated the probability that a node transmits in an arbitrary time slot, $\tau_{C C H}$, which is the input of the model. We also have the traffic density. Thus, PDR affected by hidden terminal [35] is

$$
P D R_{h}=\left\{\begin{array}{l}
1,0<d \leq \Delta L . \\
1-\left(1-\frac{\Delta L}{d}-\frac{1}{d C}\right) e^{-(R-d) C} \\
-\frac{1}{d C} e^{-(R-\Delta L) C}, \Delta L<d \leq R .
\end{array}\right.
$$


PDR impacted by concurrent collision from right-hand side nodes of the tagged node [35] is

$$
P D R_{c r}=\left\{\begin{array}{l}
e^{-\left(\beta L_{i n t}-1\right) \tau_{C C H}}\left[1-\frac{\frac{1}{\tau_{C C H}} e^{-\beta(\Delta L-d) \tau_{C C H}}}{\beta d}\right. \\
\left.+\left(1+\frac{1}{\tau_{C C H} \beta d}\right) e^{-\beta \Delta L \tau_{C C H}}\right], 0<d \leq \Delta L \\
e^{-\left(\beta L_{i n t}-1\right) \tau_{C C H}}\left[1-\left(1-\frac{\Delta L}{d}-\frac{1}{\beta d \tau_{C C H}}\right)\right. \\
\left.-\frac{\Delta L}{d}\right], \Delta L<d \leq R
\end{array}\right.
$$

PDR impacted by concurrent collision from left-hand side nodes of the tagged node [35] is

$$
\begin{array}{r}
P D R_{c l}=e^{-\beta\left(L_{i n t}-\tau_{C C H}\right)}\left[1-e^{-\beta(R-d)}\right. \\
\left.+\frac{1}{\beta d \tau_{C C H}} e^{-\beta(R-d) \tau_{C C H}}-\frac{1}{\beta d \tau_{C C H}} e^{-\beta R \tau_{C C H}}\right] .
\end{array}
$$

Hence, the final PDR of the system [35] is derived as:

$$
P D R_{\text {total }}=P D R_{h} \times P D R_{c l} \times P D R_{c r}
$$

As a result, PLR can be calculated as

$$
P L R=1-P D R_{\text {total }}
$$

\subsubsection{Synchronized Collision}

In multi-channel operations, there is a high probability for synchronized collision at the start of a channel interval among devices with ready-to-send packets. All of devices respect the 802.11 back-off rule and select a random back-off slot to avoid collision. However, in this special case, the collision can not be avoided. The main reason behind this problem 
is that the default contention window is set to 16 , which restricts each node to randomly select a back-off time slot within $\{0,1,2, \ldots, 15\}$. If there are more than 16 nodes in the network (which is a common scenario), with probability one, two nodes will select the same time slot to start transmission, thus concurrent collision happens. Moreover, any two nodes will be in the vulnerable period and thus they will still suffer from synchronized hidden terminal problem. Figure 3.4 shows the synchronized collision we observe in NS2 simulation. The MAC layer packets in the red box are transmitted almost in the same time instant, which causes synchronized collision and synchronized hidden terminal packet loss.

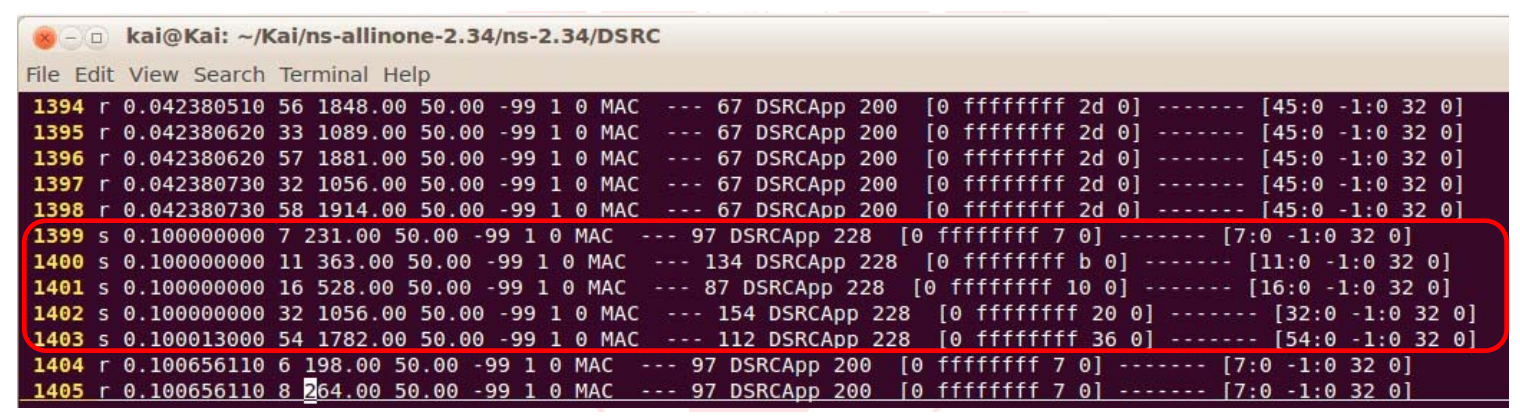

Fig. 3.3 Synchronized collision observed in NS-2 simulation.

This important impact shall be included into our analytical model.

We know the channel utilization is $\rho=\frac{\lambda_{C C H}}{\mu_{C C H}}$. And the probability that a packet is sent in a time slot is $\tau_{C C H}$. Define $P L R_{S Y N C}$ as the PLR in the first $W_{0}$ slots that mostly impacted by synchronized collision and PLR $R_{N O R M A L}$ as the PLR after the first $W_{0}$ slots that majorly impacted by interference and fading. Thus, the total packet loss in a CCHI is $P L R_{N O R M A L} \times\left(\frac{C C H I}{\sigma}-W_{0}\right) \times \tau_{C C H}+P L R_{S Y N C} \times W_{0} \times \rho$. And the total number of packets sent is $\frac{C C H I}{\sigma} \times \tau_{C C H}+W_{0} \times \rho$. Therefore, the overall packet loss rate over a CCHI 


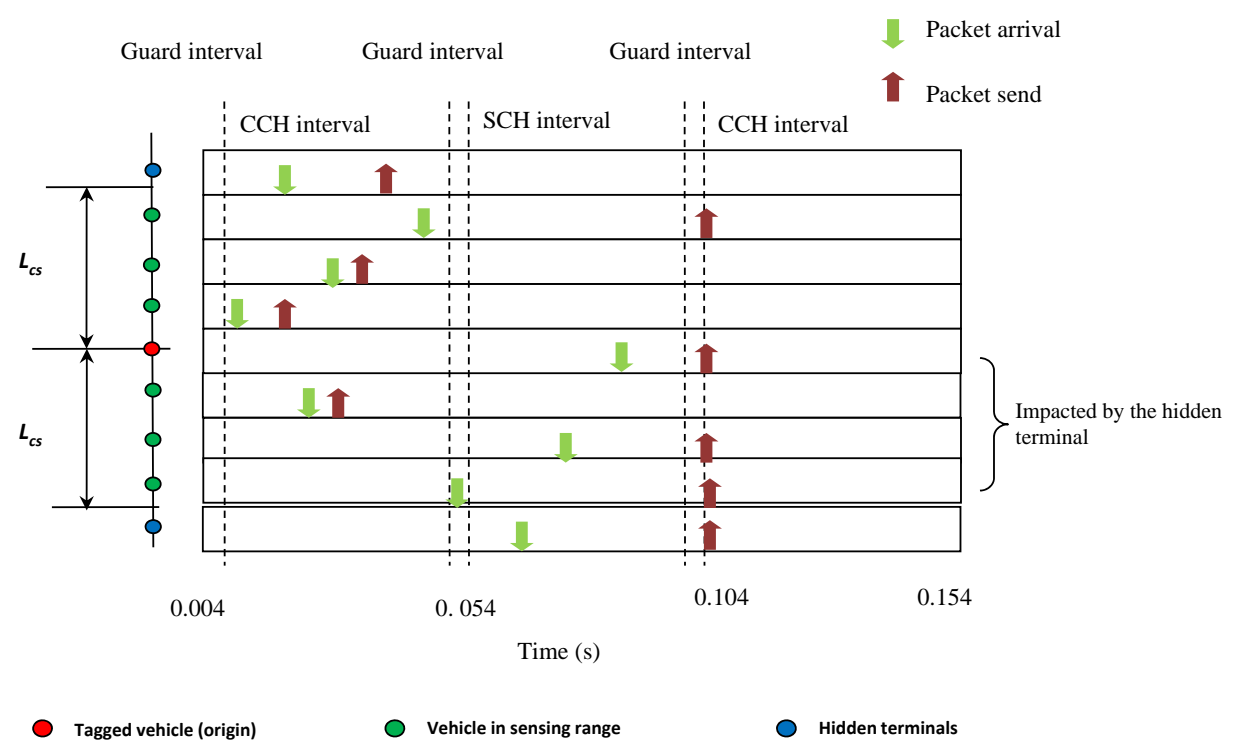

Fig. 3.4 Synchronized collision at start of a channel interval

including synchronized packet loss is

$$
\begin{aligned}
P L R_{\text {TOTAL }} & =\frac{\text { Total Packets Loss }}{\text { Total Packets Transmitted }} \\
& =\frac{P L R_{N O R M A L} \times\left(\frac{C C H I}{\sigma}-W_{0}\right) \times \tau_{C C H}+P L R_{S Y N C} \times W_{0} \times \rho}{\frac{C C H I}{\sigma} \times \tau_{C C H}+W_{0} \times \rho}
\end{aligned}
$$

where $\mathrm{N}$ is the total number of nodes within the communication range of the tagged node and $P L R_{\text {NORMAL }}$ is calculated by the normal packet arrival probability in a slot and $P L R_{S Y N C}$ is calculated using the utilization (there is at least one packet in the queue).

Therefore, the average packet loss rate of the tagged node is

$$
P L R_{A V G}=\frac{1}{N} \sum_{i} P L R_{i}
$$

where $P L R_{i}$ is the overall packet loss ratio of node i with respect to the transmissions from 
the tagged node.

\subsection{Markov Chain Model for WAVE 1609.4 Unicast in SCHs}

While in CCH, safety messages are flooded to all neighbors, non-safety services are usually transmitted upon request in unicast mode. In this mode, the sender is aware of the reception of transmitted packets. Once a transmission fails, a retransmission will be scheduled and the contention window will be doubled. Conforming to previous assumptions, we model the backoff process of the transmission as a 2-D Markov Chain with state space $\{\mathrm{s}(\mathrm{t}), \mathrm{b}(\mathrm{t})\}$, where $\mathrm{s}(\mathrm{t})$ is the backoff stage and $\mathrm{b}(\mathrm{t})$ is the backoff counter. Figure 5.6(a) presents the 2-D Markov Chain.

We know the channel utilization is $\rho=\frac{\lambda_{S C H}}{\mu_{S C H}}$. And, the probability that in an generalized time interval a node receives a packet from upper layers,

$$
P_{\text {Arrival }}^{S C H}=1-e^{-\lambda_{S C H} \sigma_{S C H}^{\prime}}
$$

where $\sigma_{S C H}^{\prime}$ is an average time slot which is

$$
\sigma_{S C H}^{\prime}=\left(1-P_{t r}\right) \sigma+P_{t r}\left(1-P_{s}\right) T_{c}+P_{t} r P_{s} T_{s}
$$

Moreover, due to channel switching and other nodes' transmission, the probability that the backoff counter gets blocked is

$$
P_{u n i}=\frac{T_{S C H I}}{T_{S Y N C}} P_{b}+\frac{T_{S Y N C}-T_{S C H I}}{T_{S Y N C}}
$$

where $T_{S Y N C}$ is the whole synchronization period. 


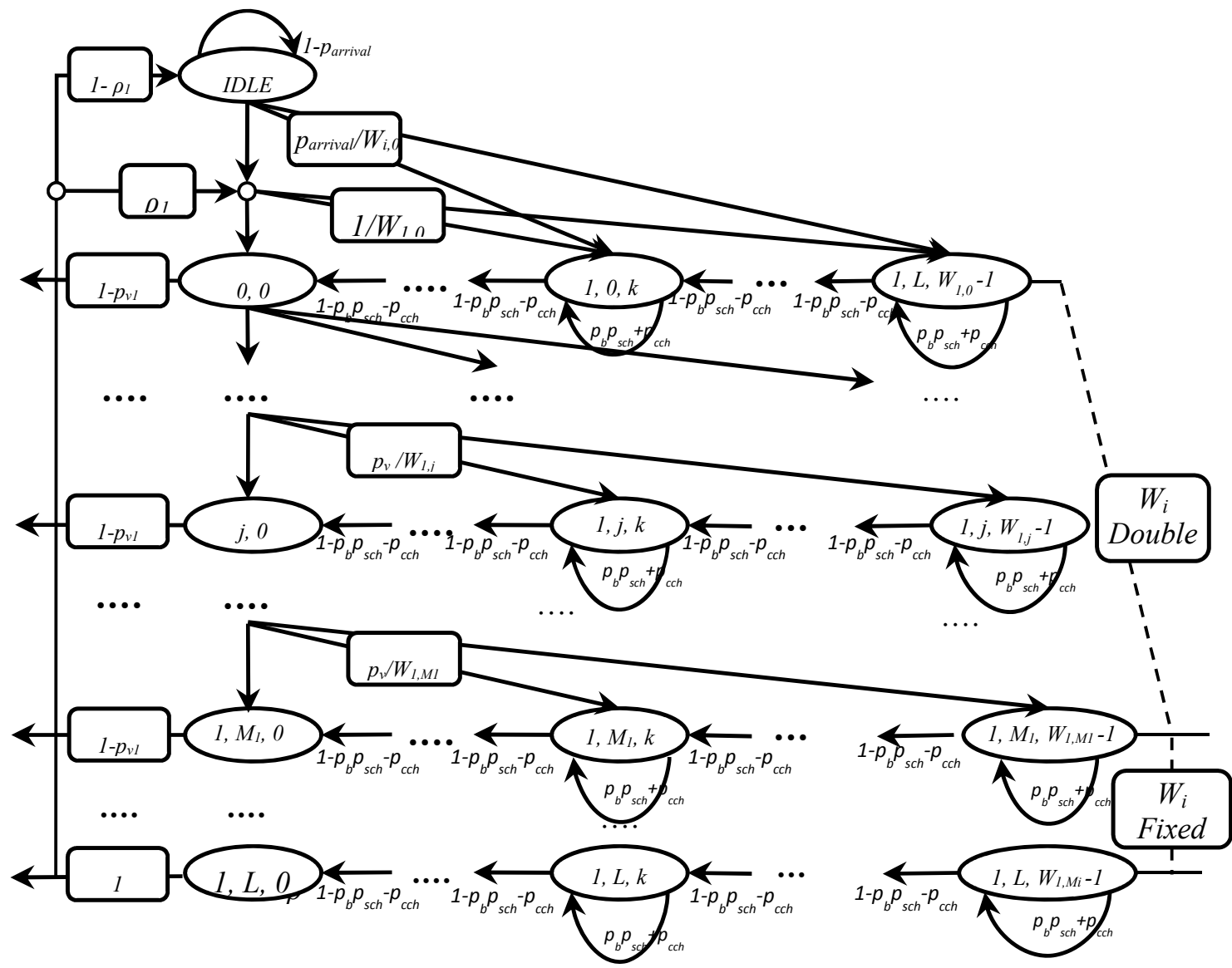

Fig. 3.5 2-D Markov Chain for Unicasting Service Channel improved over EDCA model in paper [52]

Obtain the transition probability from figure 5.6(a) and recall the fact that the sum of all stationary probabilities equals one, we are able to get the stationary state probability $\pi_{0,0}$

$$
\begin{gathered}
\pi_{0,0}=\left\{\frac{\frac{W_{0}}{2} P_{t r}\left[1-\left(2 p_{\text {uni }}\right)^{m+1}\right]}{\left(1-p_{\text {uni }}\right)\left(1-2 P_{t r}\right)}+\frac{1-P_{t r}^{m+1}}{\left(1-2 P_{t r}\right)}\right. \\
\left.+\frac{\left(1-P_{t r}^{m+1}\right)}{\left(1-P_{t r}\right)}+\frac{W_{0} / 2}{\left(1-P_{u n i}\right)}+\frac{1-\rho_{u n i}}{P_{a}}\right\}^{-1},
\end{gathered}
$$


where $P_{t r}$ is the probability that there is at least one node transmitting in current slot and is calculated as

$$
P_{t r}=1-\left(1-\tau_{S C H}\right)^{2 \beta R+1},
$$

and $T_{\text {vuln }}=\frac{\left(L_{H}+\mathbb{E}[P]\right)}{R_{d}}$ which is the vulnerable period during which the tagged nodes transmission is vulnerable to hidden terminals. The probability that a transmission is successfully completed is

$$
P_{s}=\frac{(2 \beta R+1) \tau_{S C H}\left(1-\tau_{S C H}\right)^{2 \beta R}}{P_{t r}}
$$

where $T_{s}=\frac{L_{H}+\mathbb{E}[P]}{R d}+S I F S+\sigma+A C K+D I F S+\delta . T_{s}$ is the time period for successfully transmitting a packet. And $\tau_{S C H}$ is the probability that a packet is transmitted in an arbitrary time slot

$$
\tau_{S C H}=\sum_{i=0}^{m} b_{i, 0}=\frac{1-p_{v}^{L+1}}{1-p_{v}} \pi_{0,0}
$$

Following a similar iterative method in section 3.2 .5 , we can solve $\rho_{\text {uni }}$ and $\pi_{0,0}$.

\subsubsection{System Throughput}

The normalized system throughput [9] [16] is defined as the ratio

$$
S=\frac{\mathbb{E}[\text { payload information transmitted in a slot time }]}{\mathbb{E}[\text { length of a slot time }]}
$$

which represents the portion of a unit time frame that the channel successfully transmits the payload. $P_{t r} P_{s} \mathbb{E}[P]$ is the average payload bits transmitted in a slot time. The mean 
value of a slot time is composed of three parts, namely, a fraction that the channel is idle, a fraction that the channel fails to transmit the packet due to collision and a fraction that the channel is used to successfully transmit the packet. Therefore, we can write the normalized system throughput in following form [9] [16]

$$
S=\frac{P_{t} P_{s} \mathbb{E}[P]}{\left(1-P_{t}\right) \sigma+\left(P_{t}\left(1-P_{s}\right)\right) T_{c}+P_{t} P_{s} T_{s}},
$$

where $T_{c}=\frac{L_{H}+\mathbb{E}[P]}{R d}+D I F S+\delta$ which is the time period the the backoff timer should defer when detecting an ongoing transmission.

\subsection{Tradeoff in the models}

Essentially, models of PDR in $\mathrm{CCH}$ and throughput in SCHs are functions of many parameters. However, most of them have been determined by the DSRC protocol. Suppose the traffic density information is given, the only parameter we can adjust is CCHI. Since the sum of CCHI and SCHI equals synchronization interval, which is a constant, when we increase $\mathrm{CCHI}$, the $\mathrm{SCHI}$ is decreased and vice versa. If CCHI is increased, there will be more time for transmissions in $\mathrm{CCH}$. Thus the queue utilization will be small, causing less synchronized collision. The overall PDR will increase as well. However, throughput in SCHs will decrease if CCHI is increased. From the model, we see that the less time for SCHI, the more likely SCH will be blocked. Thus less packets will be sent. This tradeoff gives us the intuition to design an algorithm that can dynamically adjust the channel interval to balance the reliability in $\mathrm{CCH}$ and throughput in SCHs. We will provide a detailed design in Chapter IV. 


\subsection{Summary}

In this chapter, we focus on the performance and reliability analysis of IEEE 1609.4 in $\mathrm{CCH}$ and $\mathrm{SCH}$ respectively. For $\mathrm{CCH}$, we leverage a 1-D Markov Chain (MC) to model broadcasting with no retransmission. In this case, we are interested the reliability of safety communications and use PLR as the reliability metric, where not only concurrent collision and hidden terminal impacts are considered but also the impact of synchronized collision is included. For SCHs, we develop a 2-D Markov Chain model to capture the features of unicasting in SCHs with retransmission. We obtain the analytical result of system throughput in this case. Moreover, this is the first work to include multi-channel operations in the analytical model under realistic assumption. This work also lays the foundation of chapter 4, where we apply these models to derive an effective multi-channel coordination algorithm to well balance the reliability and performance tradeoff in $\mathrm{CCH}$ and SCHs. 


\section{Chapter 4}

\section{Multi-Channel Coordination}

\section{Algorithm}

Ideally, $\mathrm{CCH}$ and SCHs shall be operating on different transceivers such that they do not have to share radio resources. And this is the long term goal of DSRC (10-20 years). However, according to C. Campolo [10], dual-radio devices are far more costly than singleradio devices. This leads to much longer time for DSRC to penetrate the market. Another issue for dual radio devices is that one radio would have strong interference on the other if they are physically close regardless of what channel they operate. For these reasons, single-radio devices are the main focus in the initial deployment stage of DSRC. However, the problem that the duration of $\mathrm{CCH}$ and $\mathrm{SCH}$ is halved in single-radio devices is still critical. To cope with dynamic demands of the reliability in $\mathrm{CCH}$ and throughput in SCHs, we propose a multi-channel coordination algorithm to adaptively adjust $\mathrm{CCH}$ and $\mathrm{SCH}$ intervals. The proposed algorithm maximizes SCH throughput while provisioning sufficient reliability for delay-stringent safety packets. The main idea is to set-up a lower bound of the reliability for safety messages. When traffic density is low, a very small $\mathrm{CCH}$ 
interval can fulfil this demand and the remaining time resource can contribute to non-safety services delivery. On the other hand, when the density is high, we can enlarge $\mathrm{CCH}$ interval to provision more time resource to complete the transmission of safety messages in that synchronization interval.

\subsection{The Multi-Channel Coordination Algorithm}

In this section, we propose the multi-channel coordination algorithm. We view the channel interval as limited resources for safety related messages and non-safety applications. Thus, this problem can be viewed as a Resource Optimization (RO) problem. When dispatching resources, we have to balance the reliability of time-stringent safety services and the performance of infotainment applications. We design an iterative control system to achieve this goal as figure 4.1 shows. We denote PLR as the price function in the RO problem and the Packet Delivery Ratio (PDR) is obtained as 1-PLR. Concerning our primary goal,

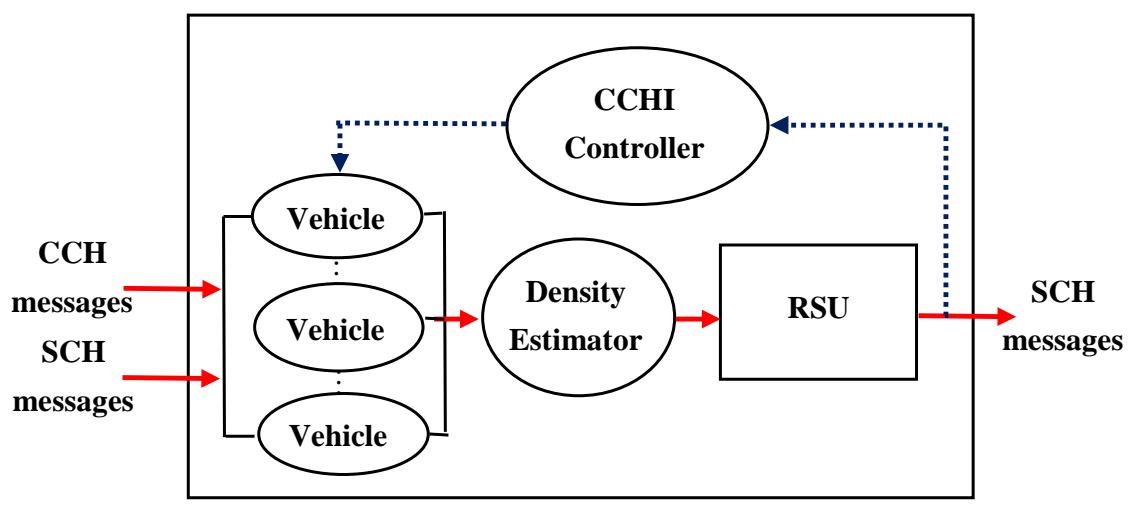

Fig. 4.1 Iterative Control System

guaranteeing the reliability of safety services, CCHI will be enlarged to relieve synchronized collision when the reliability is low, i.e. the on road traffic density is heavy. it will be reduced when the reliability is higher than the pre-defined threshold, i.e. the traffic density 
is low. The dynamics of CCHI thus is

$$
C \dot{C H} I=-\alpha_{0}\left(P D R-P D R^{t h}\right)
$$

where $\alpha_{0}$ is a constant, $\alpha_{0}>0$ and $P D R^{\text {th }}$ is a pre-defined threshold.

As a result, when CCHI increases, PDR will obviously increase as the synchronized collision is relieved. On the other hand, PDR will decrease when CCHI decreases. In summary, PDR has a positive relationship with CCHI. Dynamics of PDR is therefore

$$
P \dot{D} R=\alpha_{1}\left(C C H I-C C H I^{t h}\right)
$$

where $\alpha_{1}$ is a constant and $\alpha_{1}>0$.

With equation (4.1) and (4.2), we have

$$
P \ddot{D} R+\alpha_{0} \alpha_{1} P D R=\alpha_{0} \alpha_{1} P D R^{t h}
$$

The damp ratio in this system is 0, according to control theory [15], it exhibits nondecaying oscillations. The interval will not converge to an optimal value. To overcome this problem, a proportional-plus-derivative (PD) controller can be adopted to have a system that converges. Ergo, we have the following theorem concerning stability.

Theorem 1 (System Stability) The proposed control system is stable if $\alpha_{0}>0, \alpha_{1}>0$, $\alpha_{3}>0$ and the PD controller

$$
C C H \dot{I}(t-d)=-\alpha_{0}\left(P D R(t)-P D R^{t h}\right)-\alpha_{3} P \dot{D} R
$$

is adopted. 
Proof: The PD controller can first be constructed as follows

$$
C \dot{C H} I=\alpha_{0}\left(P D R-P D R^{t h}\right)-\alpha_{3} P \dot{D} R
$$

And due to delay, $d$, for information update in the system, the CCHI we used is actually the one at $t-d$

$$
P \dot{D} R=-\alpha_{1}\left(C C H I(t-d)-C C H I^{t h}\right) .
$$

Eventually, we have the final PD controller,

$$
C \dot{C H} I(t-d)=-\alpha_{0}\left(P D R(t)-P D R^{t h}\right)-\alpha_{3} P \dot{D} R(t) .
$$

After adopting equation (4.4), we have the new dynamic system,

$$
P \ddot{D} R+\alpha_{1} \alpha_{3} P \dot{D} R+\alpha_{0} \alpha_{1} P D R=\alpha_{0} \alpha_{1} P D R^{t h}
$$

It is easy to verify that the two characteristic roots of equation (4.8) have negative real parts, therefore the system is stable. We can finally have the PD controller by using Taylor series to expand equation (4.7) as

$$
\begin{array}{ll}
\text { CCHI } \quad(n+1-\tau) \\
=\quad S\{C C H I(n-\tau)-\lambda(P D R(n)- \\
& \left.\left.P D R^{t h}\right)-\zeta(P D R(n)-P D R(n-1))\right\}, \\
=\quad & S\left\{C C H I(n-\tau)-\lambda\left(P L R^{t h}-P L R(n)\right)\right. \\
& -\zeta(P L R(n-1)-P L R(n))\},
\end{array}
$$


where

$$
S(x)=\left\{\begin{array}{l}
0 m s, \text { if } x<0 \\
100 \mathrm{~ms}, \text { if } x>100 \\
x \mathrm{~ms}, \text { otherwise }
\end{array}\right.
$$

and $\lambda$ and $\zeta$ are step sizes that control the rate of convergence. Once we have the consensus results of the traffic density (to be discussed in the next subsection), we calculate the PLR based on previous calculated CCHI. Moreover, we can obtain the new $\mathrm{CCH}$ interval through above PD controller.

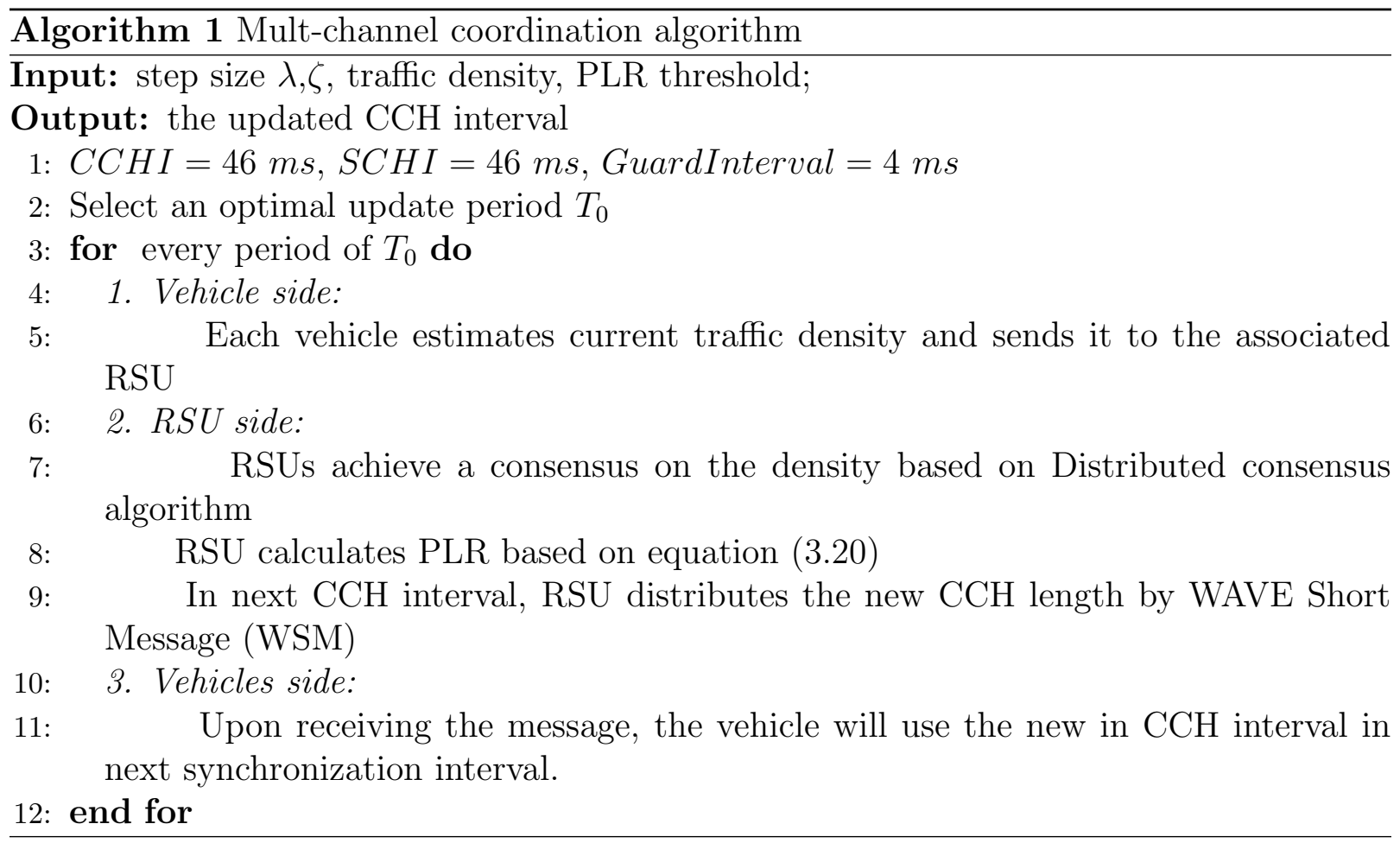




\subsection{Algorithm Convergence and Convergence Rate}

The iterative control approach is essentially a gradient descent method [23]. We want to have the PLR approach the threshold, thus the reliability is guaranteed regardless of the traffic density. According to gradient descent theory, and as we have PLR as a convex function, $\nabla$ PLR Lipschitz continuous, and appropriate step size $\zeta, \lambda$, we can guarantee a local solution [23]. Moreover, in this case, the method will eventually converge to a global desired solution. The convergence rate is dependent on the step size $\zeta, \lambda$. Large step size leads to large update each time, thus it converges faster. Conversely, small step size moves slowly to the solution but it provides higher accuracy. Thus there is a tradeoff. To achieve

the best tradeoff we shall dynamically choose the step size. In initial update stage, we choose large step size in order to move fast to the solution. When we get close, we choose to use smaller step size to avoid oscillation around the solution. In our context, we choose a small and constant step size as the range of CCHI is not large, we can approach it fast and accurately with small step size.

\subsection{Distributed consensus for estimating traffic density}

We need to estimate current traffic density as one of the inputs to our algorithm. We run a distributed consensus algorithm to gather all estimation from each single node and finally, the average value is used. Using a distributed consensus to gather the traffic density information outperforms using the estimation from a single node as it minimizes the estimation error and thus majority of nodes will benefit from the algorithm. It works as follows. Each distributed device has to estimate the density and transmit it to its connected Road Side Unit (RSU). Each RSU will calculate the mean traffic density and then deploy a distributed consensus algorithm [18] to achieve the consensus of the density. After achieving this 
consensus, they will use density as input to the proposed algorithm to produce an update interval. Finally, during next $\mathrm{CCH}$ interval, RSU will fill the update interval in a WAVE Short Message (WSM) and flood it to vehicles. As we include the new interval information in the routine WSM, it does not introduce extra message overhead in the protocol.

\subsection{Summary}

Supporting multi-channel operations in a single DSRC radio will cause significant performance loss as the bandwidth is split equally to the $\mathrm{CCH}$ and $\mathrm{SCH}$. To tackle this problem, we propose a multi-channel coordination algorithm based on the analytical model in chapter 3. This algorithm utilizes the idea of iterative control. We update the channel interval length directly based on current packet loss ratio. It converges fast to the optimal interval length and can well balance the reliability of safety messages and throughput of non-safety messages comparing to current scheme in the Standard. 


\section{Chapter 5}

\section{Model Validation and Performance}

\section{Analysis}

In this Chapter, we conduct simulations to validate the proposed analytical model and then evaluate the performance of the multi-channel coordination algorithm. We consider

a free-way system where all vehicles are distributed according to a Poisson process. Simulations are conducted on a a $2000 \mathrm{~m}$ highway segment. Each vehicle is equipped with an on-board DSRC wireless device. Road Side Units (RSUs) are also present to provide non-safety services. Hence, both V2V and V2I communications are included in the simulation. Moreover, we leverage the IEEE 802.11p as the MAC protocol and the two-ray ground reflection model as the physical propagation model. Due to finite transmission range and carrier-sense range used in our system, hidden terminals, concurrent collisions and synchronized collisions are naturally reflected in the simulations.

We begin by introducing the simulations platform in Section 5.1, and describe the parameters we use in the simulation in Section 5.2. In Section 5.3, we validate our model and in Section 5.4, we present evaluation results from the simulation. 


\subsection{Simulation Platform}

We use the Network Simulator 2 (ns-2) [39] as the main tool to simulate DSRC and the proposed algorithm. NS2 is a famous discrete event network simulator, which is publicly available under the GNU GPLv2 licence for research and development. It uses Tcl instead of MIT's Object Tcl (Otcl) as simulation scripts. The core of the program is implemented in $\mathrm{C}++$. The CMU IEEE 802.11 MAC and PHY models are used as the MAC layer and Physical layer of our system, respectively. We implement DSRC multi-channel based on

the toolkit released by Ghandour [3]. $\mathrm{CCH}$ and SCHs are interleaving as we declare one's medium to be busy when the other is operating. The system supports both broadcasting and unicasting. In addition, we use Matlab to process simulation traces.

\subsection{Simulation Setup}

We consider a typical highway scenario where the distribution of vehicles follows a Poisson distribution. Each of the nodes has the same transmission range. Especially, we choose the node in the middle of the lane as the tagged node as it does not suffer any edge effect. Each channel has a $10 \mathrm{M} \mathrm{Hz}$ bandwidth. The packet arrival processes of both $\mathrm{CCH}$ and SCHs satisfy the Poisson distribution. The packet generating rate of $\mathrm{CCH}$ is $10 \mathrm{~Hz}$ and for SCHs, the rate is $100 \mathrm{~Hz}-500 \mathrm{~Hz}$ as we consider that there are more packets from nonsafety applications. The main parameters of $802.11 \mathrm{p}$ used in the system are summarized in Table 5.1. We adopt standard two-ray ground reflection model as the physical model in the simulation. This model has been thoroughly measured and characterized in the mobile V2V environment. The model considers both the direct path and a ground reflection path. 
Table 5.1 Simulation Configuration

\begin{tabular}{|c|c|}
\hline Parameter & Value \\
\hline Density $(\beta)$ & $0.01-0.1 \mathrm{vhls} / \mathrm{m}$ \\
\hline Transmission range(R) & $450 \mathrm{~m}, 378 \mathrm{~m}, 180 \mathrm{~m}, 102 \mathrm{~m}$ \\
\hline Carrier Sense Range $\left(L_{c s}\right)$ & $750 \mathrm{~m}$ \\
\hline Interference Range $\left(L_{i n t}\right)$ & $600 \mathrm{~m}$ \\
\hline Frequency & $5.9 \mathrm{GH}$ \\
\hline PHY header $\left(P H Y_{H}\right)$ & $48 \mathrm{bits}$ \\
\hline MAC header $\left(M A C_{H}\right)$ & 28 bytes \\
\hline SIFS & $32 \mu \mathrm{s}$ \\
\hline DIFS & $64 \mu \mathrm{s}$ \\
\hline DATA rate $\left(R_{d}\right)$ & $3 \mathrm{Mbps}, 6 \mathrm{Mbps}, 12 \mathrm{Mbps}, 24 \mathrm{Mbps}$ \\
\hline Slot time $(\sigma)$ & $13 \mu s$ \\
\hline Contention Window $\left(W_{0}\right)$ & 15 \\
\hline
\end{tabular}

The received power at distance $d$ is predicted by

$$
P_{r}(d)=\frac{P_{t} G_{t} G_{r} h_{t}^{2} h_{r}^{2}}{d^{4} L}
$$

where $h_{t}$ and $h_{r}$ are the heights of the transmit and receive antennas respectively.

\subsection{Model Validation}

In this section, we validate PLR and throughput models by comparing their theoretical values with simulation results. Figure 5.1 shows the Packet Loss Ratio with varying traffic density. In this scenario, we separate the PLR caused by interference and PLR caused by fading. In Figure 5.1, the PLR only suffers from interference, which is what our model describes. When the traffic density increases, the PDR has a significant drop. The results of our model coincide with the simulation counterparts, which tell that our theoretical analysis well capture features of DSRC systems. The $5 \%$ gap between the analytical and simulation results is due to the limited precision of numerical differentiation and limited 
road range in the simulations.

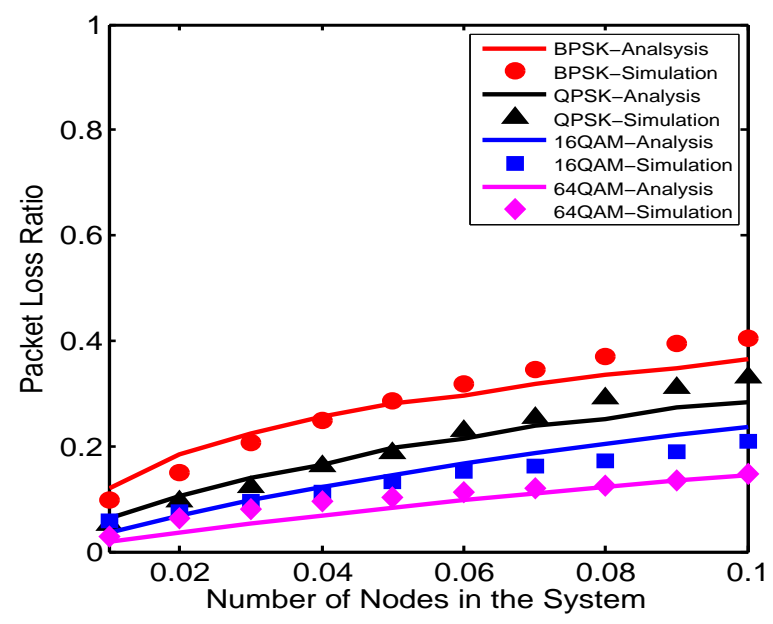

Fig. 5.1 PLR of CCH channel with BPSK and QPSK modulation

To validate the system throughput model, we perform simulations with varying packet sizes and the modulation technique is BPSK. We do not consider other modulations in this case as fading has a significant impact on them. And we can not separate throughput impacted by interference standalone and throughput impacted by fading standalone. As BPSK is resilient to fading and we place all nodes at the same place on the lane, the throughput in Figure 5.2 is only impacted by interference, which is also the focus of our throughput model for MAC layer. We observe the throughput in SCHs for different packet sizes in Figure 5.2. The throughput has a negative relation with the traffic density as interference will be stronger when more nodes are present. The simulation results also match well with the proposed model. When setting the packet size to 200 bytes, the throughput is slightly smaller. This is because the network condition is not saturated. 


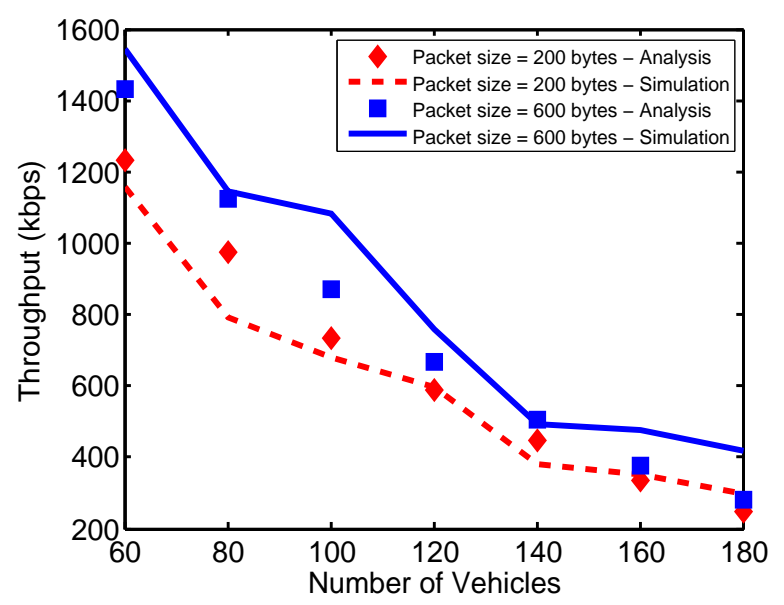

Fig. 5.2 Throughput in SCHs for different packet sizes

\subsection{Performance Evaluation}

We test the proposed algorithm with step sizes $\lambda$ and $\zeta$ equal to 0.02 and 0.01 respectively.

We take the BPSK communication range as the reference range when calculating PLR.

\subsubsection{Impacts of Main Parameters on the Performance Synchronized Collision Problem}

We demonstrate the synchronized collision in this experiment. We focus on $\mathrm{CCH}$ and divide $\mathrm{CCH}$ interval into five time slices. The first time slice is $0 \mathrm{~ms}-5 \mathrm{~ms}$, which suffers synchronized collision most. The second slice is $5 \mathrm{~ms}-10 \mathrm{~ms}$. For other slices, the length is $10 \mathrm{~ms}$. Then we record number of packets transmitted during each time slice. Figure 5.3 shows the percentage of total packets transmitted during each time slice. As we observe, the number of packets transmitted in the first slice is far more than number of packets transmitted in other slices. The reason is that each time, packets which have been generated but not able to be transmitted in time will be put in the queue. In the next synchronization 


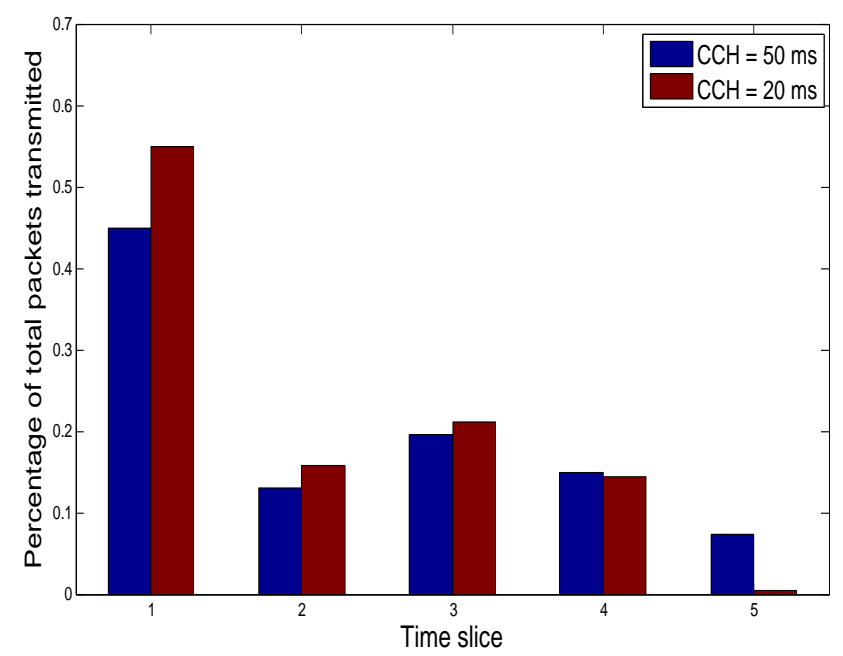

Fig. 5.3 Percentage of packets sent during each time slice (Traffic density $=$ $0.4 \mathrm{vhls} / \mathrm{m})$

interval, these packets are ready to be transmitted right away. Almost each node has a packet ready to be transmitted at the same time (the start of the interval). Thus most packets are sent in the first time slice. This is also the reason for synchronized collision as they may very likely to choose the same time slot to start the backoff process. Thus PLR of the system is thus degraded.

\section{Impact of packet size on PLR}

The relationship between the overall PLR and packet size with QPSK modulation is shown in Figure 5.4. PLR increases with packet size. This is because the larger the packets, the longer it takes to be transmitted, thus leading to longer vulnerable period.

\section{Impact of traffic density and modulation techniques on PLR}

Figure 5.5 shows the the impact of modulation techniques and traffic density on PLR. It is shown that 16QAM and 64 QAM always suffer high packet loss than BPSK and QPSK. 


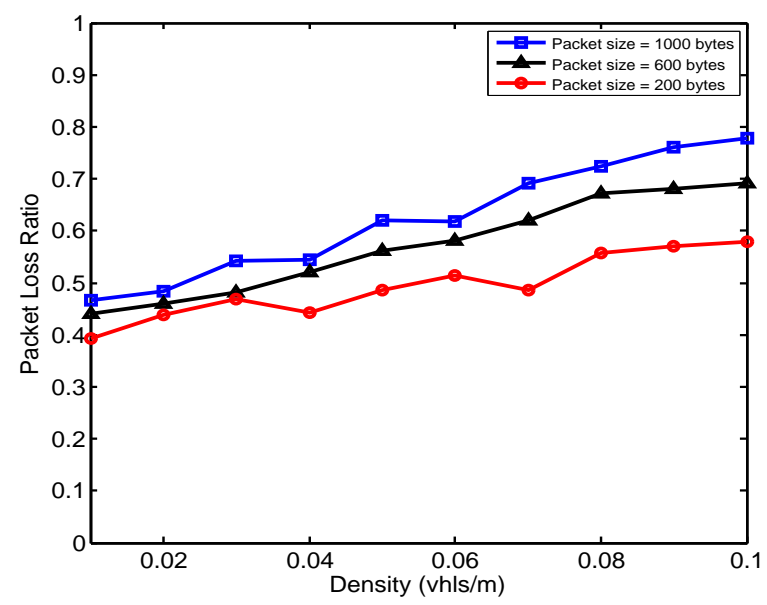

Fig. 5.4 Impact of packet size and traffic density on Packet Loss Ratio in $\mathrm{SCHs}$

Moreover, BPSK outperforms other modulation techniques in low density and QPSK is the best in high density. We further analyse the PLR by showing the portion caused by interference $\left(P L R_{\text {int }}\right)$ and the portion caused by physical fading $\left(P L R_{\text {fading }}\right)$. Figure 5.5(b) shows $P L R_{\text {int }}$ increases with traffic density and data rate. This is due to the fact that higher data rate reduces the vulnerable period, and thus suffers less hidden terminal impact. Nevertheless, Figure 5.5(c) shows that high level modulations incurs high PLR fading as they have comparatively short transmission range. Any receiver beyond that range will not receive packets. As $P L R_{\text {int }}$ and $P L R_{\text {fading }}$ have inverse trend when density increases, there is a cross point in total PLR for BPSK and QPSK. As in low density, the interference is not that high, and BPSK is more resilient to fading, so BPSK has a lower total PLR. However, in high density, interference is more significant, which makes QPSK outperforms BPSK. 


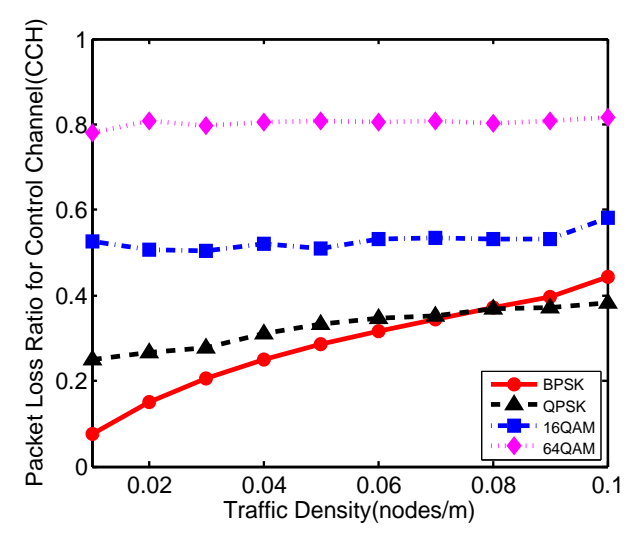

(a) Total PLR

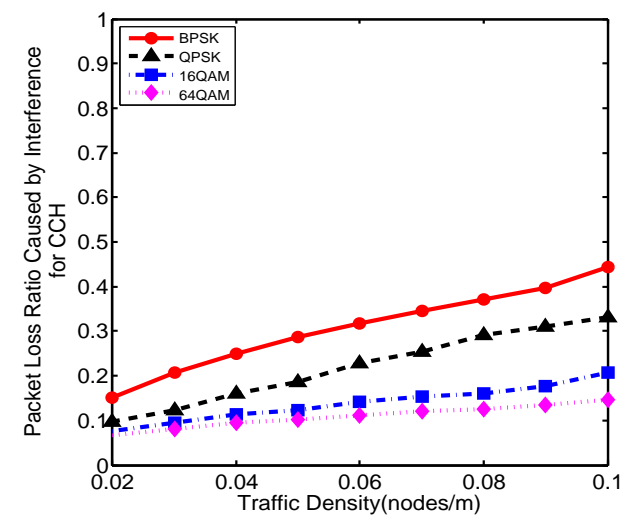

(b) PLR caused by inteference

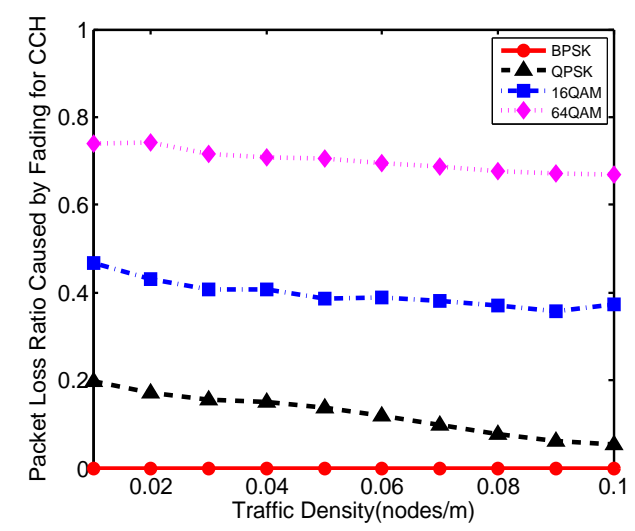

(c) PLR caused by fading

Fig. 5.5 Impact of traffic density on DSRC reliability

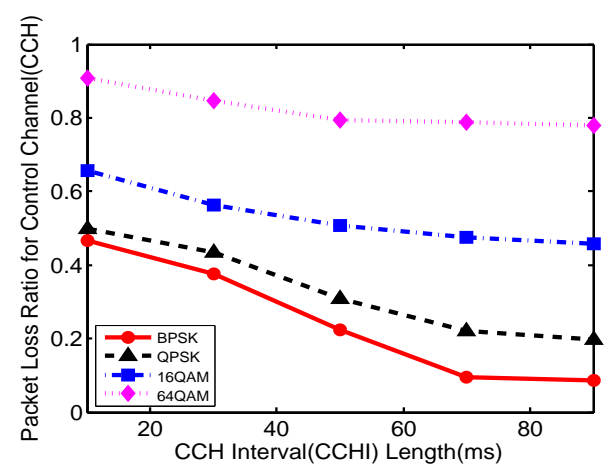

(a) Total PLR

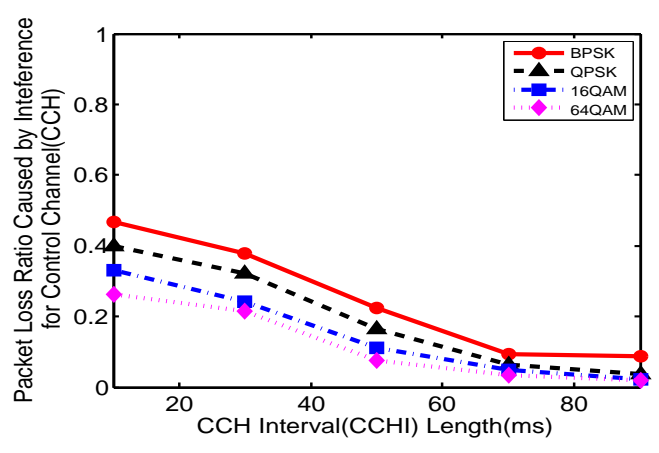

(b) PLR caused by Inteference

Fig. 5.6 Impact of $\mathrm{CCH}$ interval on DSRC reliability 


\section{Impact of $\mathrm{CCH}$ interval on saturated throughput}

Figure 5.6 and Figure 5.7 show the impact of CCH interval on PLR and saturated throughput of the tagged node. From the figure, we observe that larger CCHI can reduce PLR, thus increasing reliability. While for SCHs, if the CCHI increases, SCHI would correspondingly decrease and vice versa. This results in a decrease in the saturated throughput in SCHs.

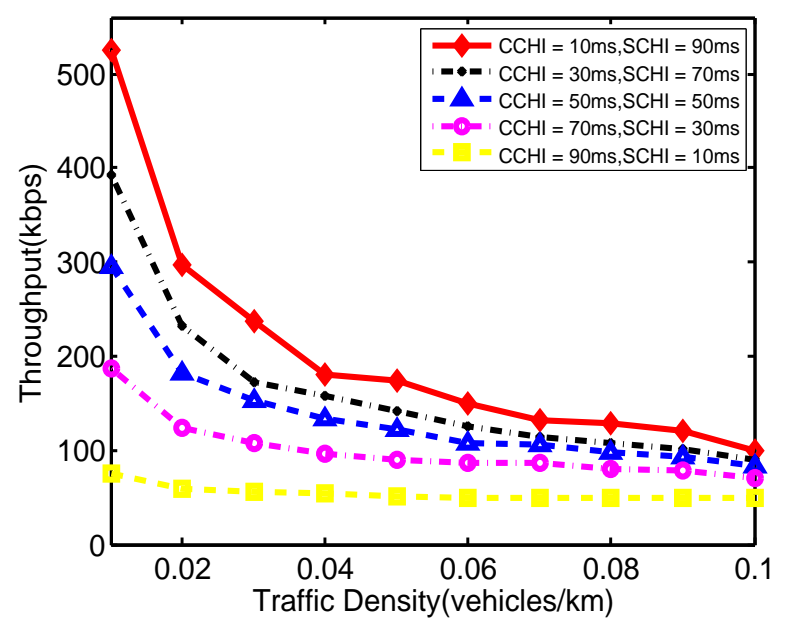

Fig. 5.7 Impact of traffic density and $\mathrm{CCH}$ interval (CCHI) on Saturated Throughput in SCHs

\subsubsection{Performance of the proposed algorithm}

We compare the performance of our algorithm with that of IEEE 1609.4 alternating access.

We set the desired PLR to 0.2 , a reasonably high reliability in automotive industry [13]. From Figure 5.8, we observe that optimal $\mathrm{CCH}$ interval increases with traffic density. As mentioned before, high traffic density leads to poor reliability as more inter-vehicle interference appears. We should enlarge $\mathrm{CCH}$ interval to provide more reliability. To this purpose, the proposed algorithm responds quickly to the PLR change and accurately adjusts $\mathrm{CCH}$ interval. In terms of accuracy, we mean that the algorithm constrains the 
PLR under the pre-defined reliability requirement, and then provisions all the bandwidth to SCHs, thus increasing SCH throughput. Figure 5.9 presents the PLR varies with traffic density under the optimal interval. The proposed algorithm under all densities keeps the PLR under the desired reliability. Our algorithm significantly outperforms the alternating

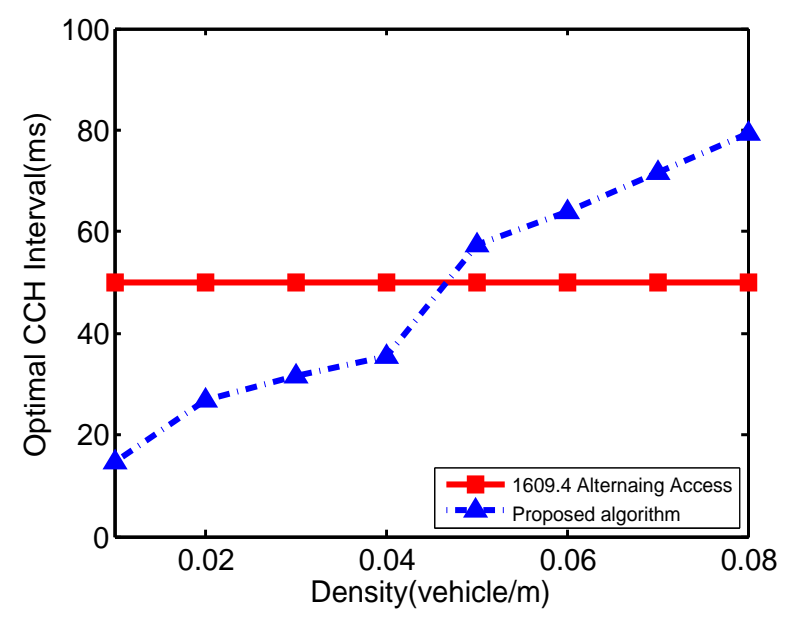

Fig. 5.8 Optimal CCH interval length

access, which has around $40 \%$ packet loss when the density is around 0.08 vehicles $/ \mathrm{m}$. Figure 5.10 shows the throughput in SCHs of the tagged node varies with traffic density. When the traffic density is low (i.e. under 0.05 vehicle $/ \mathrm{m}$ ) the proposed algorithm leads to the highest throughput possible in this scenario as the smallest possible $\mathrm{CCH}$ interval is used and more operating interval is reserved for $\mathrm{SCH}$. When the density is high, $\mathrm{CCH}$ interval is enlarged to provide more reliability. Additionally, we find that in low density cases, the proposed algorithm achieves significantly higher SCH throughput than the other while it only has minor throughput difference under high density. It is believed that our algorithm is lightweight while effective. It is flexible to add different messages in our algorithm, e.g. WSA and RFS. In some research, WSA and RFS are sent in every duty cycle. However, some work just use WSA and RFS as handover message. Our algorithm 


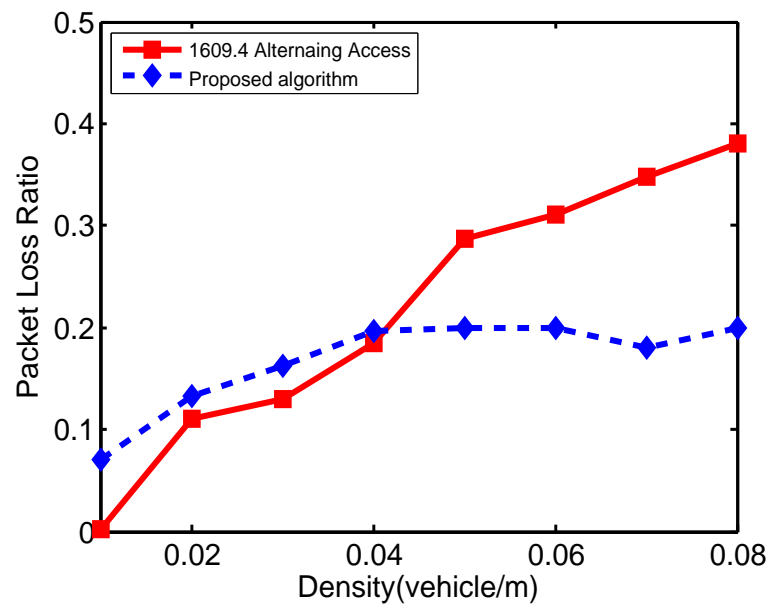

Fig. 5.9 Packet Loss Ratio in $\mathrm{CCH}$ under the desired interval

works in both scenarios as we directly control the reliability which is more flexible in design and implementation comparing to other algorithms.

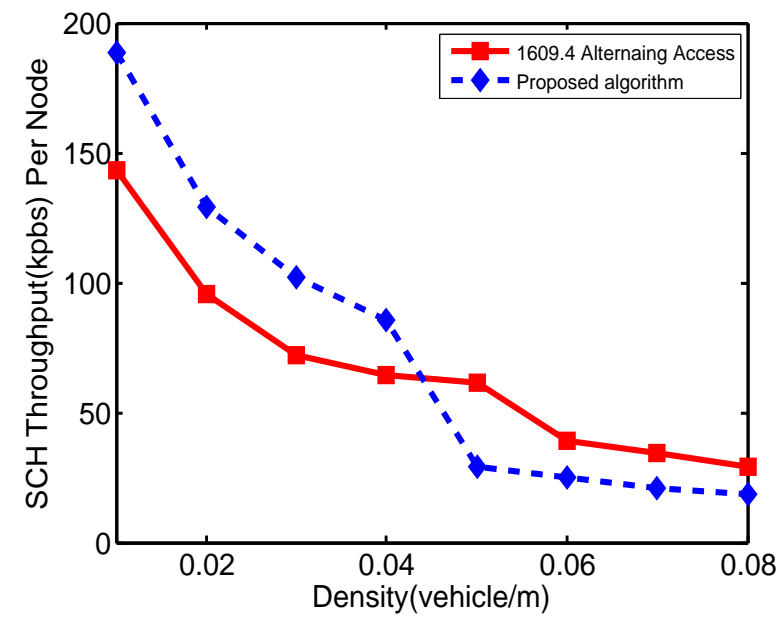

Fig. 5.10 Throughput in SCHs under the desired interval 


\subsection{Summary}

In this chapter, we first introduce our simulation platform and then describe the set-up of our system. The analytical model is validated by comparing its theoretical values and the simulation results. Moreover, results also show that our algorithm relieves synchronized collision and is effective to guarantee safety message reliability as well as achieving high throughput in low density scenario. 


\section{Chapter 6}

\section{Conclusions and Future Directions}

This thesis addresses a dominating problem for DSRC commercialization that how much DSRC can be used to support non-safety services while providing sufficient reliability for safety applications. Though the primary purpose of DSRC is to provide reliable communication for safety related applications, non-safety services also play a major role in DSRC's deployment as it makes DSRC more cost-effective. Control channel and service channels operate on a TDMA basis. Therefore, how to divide the time interval to achieve the requirements in both channels is a critical issue. To tackle this challenge, we first build two analytical models for $\mathrm{CCH}$ broadcasting and $\mathrm{SCH}$ unicasting based on Markov Chain (MC), where multi-channel operation is included and synchronized collision is analysed. In addition, we propose a multi-channel coordination algorithm to adaptively choose an optimal CCH interval based on current Packet Loss Ratio (PLR). A PLR threshold is predefined. If current PLR exceeds the threshold, the $\mathrm{CCH}$ interval will be updated to a larger value to guarantee the reliability. Otherwise, it will be reduced to provide more bandwidth for SCHs. Our approach converges rapidly to the optimal interval. Moreover, if the traffic density remains stable, the algorithm quickly converges to the optimal channel interval. 
We hope to shed insights on how to design an effective channel access approach to increase SCH throughput, and thus, expediting DSRC's deployment.

\subsection{Future Directions}

In addition to simulation work in NS2, we also plan to test this method with real DSRC radios. Moreover, data from real-world experiments would help build more accurate analytical model, e.g. we can use machine learning methods to find a better propagation model. It is also interesting if we could design a distributed multi-channel coordination algorithm that is independent of RSUs while having channel synchronization. Another possible direction is to study DSRC vehicle-to-motorcycle (V2M) and vehicle-to-pedestrian (V2P) communication. Figure 6.1 is a demo by Honda to demonstrate their new DSRC

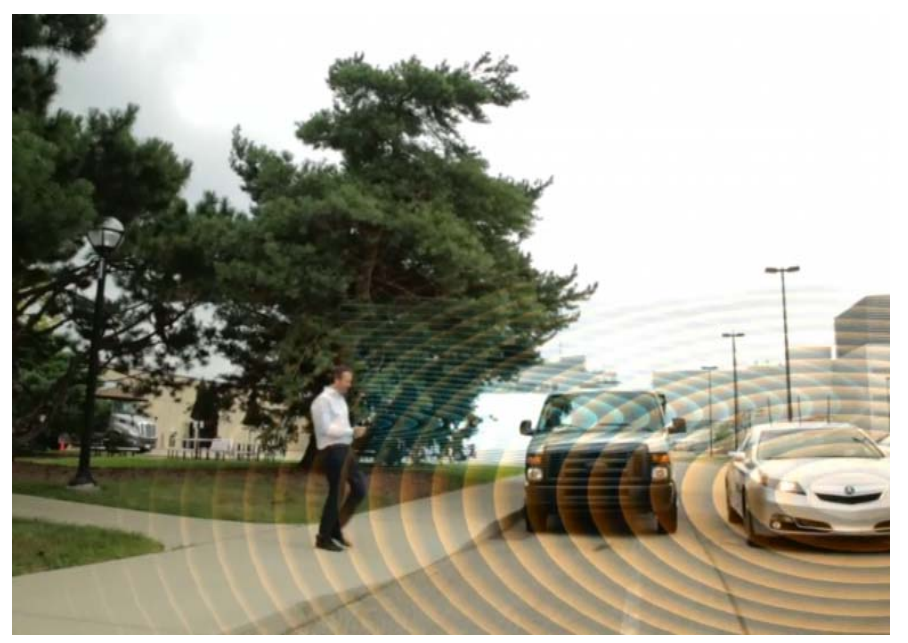

Fig. 6.1 DSRC on-board unit can also communicate with smart-phone equipped pedestrians [32]

V2P systems. These new contexts bring out new challenging problems. As the density of people, especially in downtown area is larger than that of vehicles, this will challenge traditional congestion control techniques. Another challenge is how cellphone can support 
DSRC without significant battery cost. Figure 6.2 is a primary investigation of this problem by Qualcomm. They have integrated DSRC hardware support to their new generation phones. To save battery, a possible approach would be to design an intelligent mode switch algorithm that enables cellphone to be aware of when to wake up to receive safety messages.

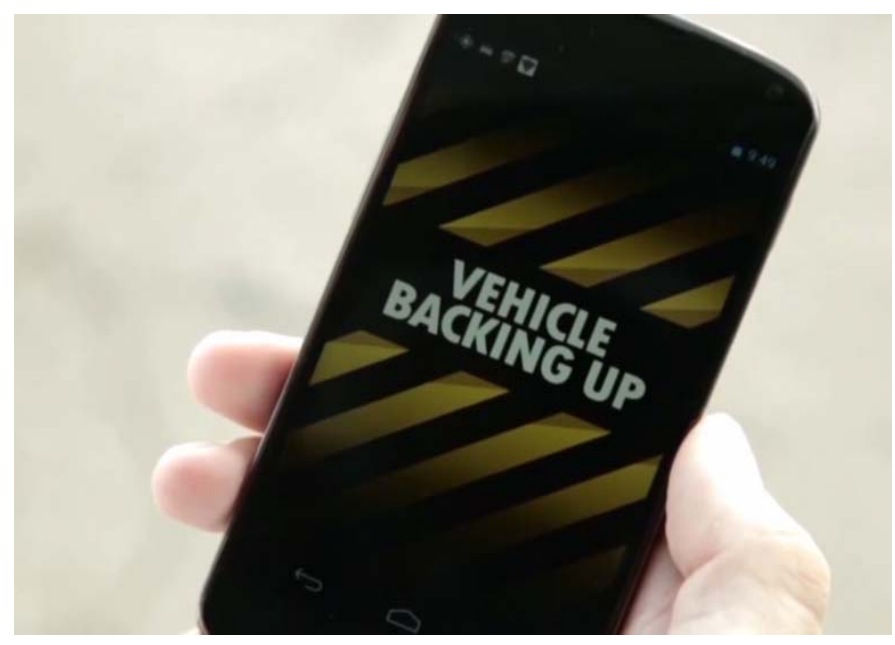

Fig. 6.2 DSRC enabled smart-phone [32] 


\section{References}

[1] Geoff Gordon, Gradient descent revisited, http : //www.cs.cmu.edu/ggordon/10725F12/slides/05 - gd-revisited.pdf, 2010.

[2] GM Develops Mobile Technology That Watches Road Ahead Portable devices debut at Intelligent Transport Systems World Congress. http://media.gm.com/media/us/en, 2011.

[3] IEEE Standard for Wireless Access in Vehicular Environments (WAVE)-Multichannel Operation. IEEE Std 1609.4-2010 (Revision of IEEE Std 1609.4-2006), pages $1-89,2011$.

[4] Ghandour Ali, Marco Di Felice, Luciano Bononi, and Hassan Artail. Modeling and Simulation of WAVE 1609.4-based Multi-channel Vehicular Ad Hoc Networks. In Proceedings of the 5th International ICST Conference on Simulation Tools and Techniques, pages 148-156, ICST, Brussels, Belgium, Belgium, 2012.

[5] Marica Amadeo, Claudia Campolo, and Antonella Molinaro. Enhancing IEEE 802.11p/WAVE to provide infotainment applications in VANETs. Ad Hoc Networks, 10(2):253-269, March 2012.

[6] Association for Safe International Road Travel, http://www.asirt.org/.

[7] Fan Bai and Bhaskar Krishnamachari. Spatio-temporal Variations of Vehicle Traffic in VANETs: Facts and Implications. In Proceedings of the Sixth ACM International Workshop on VehiculAr InterNETworking, VANET 09, pages 43-52, New York, NY, USA, 2009. ACM.

[8] Fan Bai and Hariharan Krishnan. Reliability Analysis of DSRC Wireless Communication for Vehicle Safety Applications. In Intelligent Transportation Systems Conference, 2006, pages 355-362, 2006.

[9] Lotfi Benmohamed and Semyon M. Meerkov. Feedback control of congestion in packet switching networks: the case of a single congested node. ACM Transactions on Networking, 1(6):693-708, 1993. 
[10] Giuseppe Bianchi. Performance analysis of the IEEE 802.11 distributed coordination function. IEEE Journal on Selected Areas in Communications, 18(3):535-547, September 2006.

[11] Claudia Campolo and Antonella Molinaro. Multichannel communications in vehicular Ad Hoc networks: a survey. IEEE Communications Magazine, 51(5):158-169, 2013.

[12] Claudia Campolo, Antonella Molinaro, Alexey Vinel, and Yan Zhang. Modeling Prioritized Broadcasting in Multichannel Vehicular Networks. IEEE Transactions on Vehicular Technology, 61(2):687-701, 2012.

[13] Charles Murray. Slideshow: Smarter Cars Paving Road to Intelligent Highways. http://www.designnews.com/, 2012.

[14] Qi Chen, Daniel Jiang, and Luca Delgrossi. IEEE 1609.4 DSRC multi-channel operations and its implications on vehicle safety communications. In Vehicular Networking Conference (VNC), 2009 IEEE, pages 1-8, 2009.

[15] Rahim Tafazolli Chong Han, Mehrdad Dianati and Ralf Kernchen. Asynchronous Multi-Channel MAC for Vehicular Ad Hoc Networks. In Vehicular Networking Conference (VNC), 2011 IEEE, pages 109-115, 2011.

[16] Damping ratio,. http://en.wikipedia.org/wiki/Damping_ratio, 2010.

[17] Fred Daneshgaran, Massimiliano Laddomada, Fabio Mesiti, and Marina Mondin. Unsaturated throughput analysis of ieee 802.11 in presence of non ideal transmission channel and capture effects. IEEE Transactions on Wireless Communications, 7(4):12761286, April 2008.

[18] Andreas Meier Wieland Holfelder Daniel Jiang, Vikas Taliwal. Design of 5.9 ghz dsrcbased vehicular safety communication. IEEE Wireless Communications, 13(5):36-43, 2006 .

[19] Distributed Consensus,. http : //en.wikipedia.org/wiki/Consensus_(computer_science), 2010.

[20] Stephan Eichler. Performance Evaluation of the IEEE 802.11p WAVE Communication Standard. In 2007 IEEE 66th Vehicular Technology Conference, 2007. VTC-2007 Fall., pages 2199-2203, 2007.

[21] FCC. Amendment of the Commission's Rules Regarding Dedicated Short-Range Communication Services in the 5.850-5.925 GHz band (5.9 GHz band). FCC Memorandum Opinion and Order, 99(PrePrints), 2006. 
[22] Daniel Gerlough. Use of Poisson Distribution in Highway Traffic. In Eno Foundation for Highway Traffic Control, Saugatuck, Connecticut, 1955.

[23] Gradient descent,. http ://en.wikipedia.org/wiki/Gradient_descent.

[24] Wenbo He, Xue Liu, and Klara Nahrstedt. A feedback control scheme for resource allocation in wireless multi-hop ad hoc networks. In Proceedings of the The Second Annual International Conference on Mobile and Ubiquitous Systems: Networking and Services.

[25] Tom H.Luan, Xuemin(Sherman) Shen, and Fan Bai. Integrity-Oriented Content Transmission in Highway Vehicular Ad Hoc Networks. In 2013 Proceedings IEEE INFOCOM, pages 1-9, 2013.

[26] Kezhu Hong, John B. Kenney, Vinuth Rai, and Kenneth P. Laberteaux. Evaluation of Multi-Channel Schemes for Vehicular Safety Communications. In 2010 IEEE 71st Vehicular Technology Conference (VTC 2010-Spring), pages 1-5, 2010.

[27] Mahdi Jalili. A simple consensus algorithm for distributed averaging in random geographical networks. Pramana, 79(3):493-499, 2012.

[28] Daniel Jiang and Luca Delgrossi. IEEE 802.11p: Towards an international standard for wireless access in vehicular environments. In Vehicular Technology Conference, 2008. VTC Spring 2008. IEEE, pages 2036-2040, 2008.

[29] Arthur Carter John Harding, Gregory Davis. Cooperative Intersection Collision Avoidance Systems (CICAS), USDOT Major Initiative. http://www.its.dot.gov/cicas/index.htm.

[30] John Kenney. Dedicated Short-Range Communications (DSRC) Standards in the United States. Proceedings of the IEEE, 99(7):1162-1182, 2011.

[31] Kai Liu, Jinhua Guo, Ning Lu, and Fuqiang Liu. RAMC: A RSU-Assisted MultiChannel Coordination MAC Protocol for VANET. In 2009 IEEE GLOBECOM Workshops, pages 1-6, 2009.

[32] Loz Blain. Honda testing vehicle-to-vehicle communications to eliminate SMIDSY accidents. http://www.gizmag.com/honda-dsrc-smidsy-motorcycle/28929/pictures, 2011.

[33] Xiaomin Ma and Xianbo Chen. Delay and Broadcast Reception Rates of Highway Safety Applications in Vehicular Ad Hoc Networks. In 2007 Mobile Networking for Vehicular Environments, pages 85-90, 2007. 
[34] Xiaomin Ma, Xianbo Chen, and Hazem H. Refai. Performance and reliability of DSRC vehicular safety communication: a formal analysis. EURASIP J. Wirel. Commun. Netw., 2009:3:1-3:13, January 2009.

[35] Xiaomin Ma, Jinsong Zhang, and Tong Wu. Reliability Analysis of One-Hop SafetyCritical Broadcast Services in VANETs. IEEE Transactions on Vehicular Technology, 60(8):3933-3946, 2011.

[36] Tony K. Mak, Kenneth P. Laberteaux, Raja Sengupta, and Mustafa Ergen. Multichannel Medium Access Control for Dedicated Short-Range Communications. IEEE Transactions on Vehicular Technology, 58(1):349-366, 2009.

[37] Hassan Artail Luciano Bononi Marco Di Felice, Ali J. Ghandour. On the Impact of Multi-Channel Technology on Safety-Message Delivery in IEEE 802.11p/1609.4 Vehicular Networks. In 2012 21st International Conference on Computer Communications and Networks (ICCCN), pages 1-8, 2012.

[38] Georgios Karagiannis Martijn van Eenennaam, Anne van de Venis. Impact of IEEE 1609.4 channel switching on the IEEE 802.11p beaconing performance. In Wireless Days (WD), 2012 IFIP, pages 1-8, 2012.

[39] Steve McCanne and Sally Floyd. ns Network Simulator. http://www.isi.edu/nsnam/ns/.

[40] Jelena. Misic, Ghada Badawy, and Vojislav B. Misic. Performance Characterization for IEEE 802.11p Network With Single Channel Devices. IEEE Transactions on Vehicular Technology, 60(4):1775-1787, 2011.

[41] Jelena Misic, Ghada Badawy, Saeed Rashwand, and Vojislav B. Misic. Tradeoff Issues for CCH/SCH Duty Cycle for IEEE 802.11p Single Channel Devices. In 2010 IEEE Global Telecommunications Conference (GLOBECOM 2010), pages 1-6, 2010.

[42] Jorg Ott and Dirk Kutscher. Drive-thru Internet: IEEE 802.11b for "automobile" users. In INFOCOM 2004. Twenty-third AnnualJoint Conference of the IEEE Computer and Communications Societies, volume 1, pages -373, 2004.

[43] Pete Goldin. Pioneering IntelliDrive technologies in Michigan. http://www.itsinternational.com/, 2010.

[44] Constantin Scheuermann Robert Lasowski, Florian Gschwandtner and Markus Duchon. A Multi Channel Synchronization Approach in Dual Radio Vehicular Ad-Hoc Networks. In 2011 IEEE 73rd Vehicular Technology Conference (VTC Spring), pages $1-5,2011$. 
[45] Wee Lum Tan, Wing Cheong Lau, and OnChing Yue. Modeling resource sharing for a road-side access point supporting drive-thru internet. In Proceedings of the sixth ACM international workshop on VehiculAr InterNETworking, VANET '09, pages 3342, New York, NY, USA, 2009. ACM.

[46] The CAMP Vehicle Safety Communications Consortium consisting of BMW, DaimlerChrysler, Ford, GM, Nissan, Toyota, and VW. Vehicle Safety Communications Project Final Report. volume 1, pages $-373,2006$.

[47] U.S. Department of Transportation. Dedicated Short Range Communications Frequently Asked Questions.

[48] Qing Wang, Supeng Leng, Huirong Fu, and Yan Zhang. An IEEE 802.11p-Based Multichannel MAC Scheme With Channel Coordination for Vehicular Ad Hoc Networks. IEEE Transactions on Intelligent Transportation Systems, 13(2):449-458, 2012.

[49] Zhe Wang and Mahbub Hassan. How much of DSRC is available for non-safety use? In Proceedings of the fifth ACM international workshop on VehiculAr Inter-NETworking, VANET '08, pages 23-29, New York, NY, USA, 2008. ACM.

[50] Qing Xu, Tony Mak, Jeff Ko, and Raja Sengupta. Vehicle-to-Vehicle Safety Messaging in DSRC. In In Proceedings of the 1st ACM Workshop on Vehicular Ad hoc Networks (VANET 04), 2004.

[51] Yuan Yao, Lei Rao, Xue Liu, and Xingshe Zhou. Performance and Reliability Analysis of IEEE 802.11p Safety Communication in a Highway Environment. volume 62, pages 1591-1599, 2013.

[52] Yuan Yao, Lei Rao, Xue Liu, and Xingshe Zhou. Delay Analysis and Study of IEEE 802.11p based DSRC Safety Communication in a Highway Environment. In 2013 Proceedings IEEE INFOCOM, pages 1591-1599, 2013.

[53] Xiaoyan Yin, Xiaomin Ma, and Kishor S. Trivedi. Performance of BSM Dissemination in Multichannel DSRC. Vehicular Technology Conferences, 99(PrePrints), 2013.

[54] Xiaoyan Yin, Xiaomin Ma, Kishor S. Trivedi, and Alexey Vinel. Performance and Reliability Evaluation of BSM Broadcasting in DSRC with Multi-channel Schemes. IEEE Transactions on Computers, 99(PrePrints):1, 2013. 